\title{
Symmetric embeddings of free lattices into each other
}

\author{
Gábor Czédli, Gergő Gyenizse and Ádám Kunos
}

Dedicated to Ralph Freese and J. B. Nation on their seventieth birthdays.

\begin{abstract}
By a 1941 result of $\mathrm{Ph}$. M. Whitman, the free lattice $\mathrm{FL}(3)$ on three generators includes a sublattice $S$ that is isomorphic to the lattice $\mathrm{FL}(\omega)=\mathrm{FL}\left(\aleph_{0}\right)$ generated freely by denumerably many elements. The first author has recently "symmetrized" this classical result by constructing a sublattice $S \cong \mathrm{FL}(\omega)$ of $\mathrm{FL}(3)$ such that $S$ is selfdually positioned in $\mathrm{FL}(3)$ in the sense that it is invariant under the natural dual automorphism of FL(3) that keeps each of the three free generators fixed. Now we move to the furthest in terms of symmetry by constructing a selfdually positioned sublattice $S \cong \mathrm{FL}(\omega)$ of $\mathrm{FL}(3)$ such that every element of $S$ is fixed by all automorphisms of FL(3). That is, in our terminology, we embed $\mathrm{FL}(\omega)$ into $\mathrm{FL}(3)$ in a totally symmetric way. Our main result determines all pairs $(\kappa, \lambda)$ of cardinals greater than 2 such that $\mathrm{FL}(\kappa)$ is embeddable into $\mathrm{FL}(\lambda)$ in a totally symmetric way. Also, we relax the stipulations on $S \cong \mathrm{FL}(\kappa)$ by requiring only that $S$ is closed with respect to the automorphisms of $\operatorname{FL}(\lambda)$, or $S$ is selfdually positioned and closed with respect to the automorphisms; we determine the corresponding pairs $(\kappa, \lambda)$ even in these two cases. We reaffirm some of our calculations with a computer program developed by the first author. This program is for the word problem of free lattices, it runs under Windows, and it is freely available.
\end{abstract}

Mathematics Subject Classification. 06B25.

Keywords. Free lattice, sublattice, Dual automorphism, Symmetric embedding, Selfdually positioned, Totally symmetric embedding, Lattice word problem, Whitman's condition, FL(3), FL(omega).

Presented by P. Jipsen.

This research was supported by the National Research, Development and Innovation Fund of Hungary under the KH 126581 funding scheme. 


\section{Introduction and our results}

There are many nice and deep results on free lattices of the variety of all lattices. A large part of these results were achieved by Ralph Freese and J. B. Nation, to whom this paper is dedicated. Some of these results are included in $[7,8,10,11,14,15]$ and in the monograph Freese, Ježek, and Nation [9], but this list is far from being complete. The monograph just mentioned serves as the reference book for the present paper.

By a classical result of Whitman [20], the free lattice $\operatorname{FL}(\omega)=\operatorname{FL}\left(\aleph_{0}\right)$ on denumerably many free generators is isomorphic to a sublattice of the free lattice FL(3) with three free generators. In fact, we know from a deep result of Tschantz [19] that there are many copies of $\mathrm{FL}(\omega)$ in $\mathrm{FL}(3)$; namely, every infinite interval of $\mathrm{FL}(3)$ includes a sublattice isomorphic to $\mathrm{FL}(\omega)$. For more about free lattices, the reader is referred to Freese, Ježek and Nation [9]. In this paper, we embed free lattices into each other symmetrically. For a free lattice $F$,

$\delta=\delta_{F}$ will denote the natural dual automorphism of $F$ that keeps the free generators fixed;

it is uniquely determined. A subset (or a sublattice) $S$ of $F$ is selfdually positioned if $\delta(S)=S$. Selfduality is a sort of symmetry, and a selfdually positioned sublattice is necessarily selfdual. As the main result of [2], the first author proved that

FL(3) has a selfdually positioned sublattice that is isomorphic to $\mathrm{FL}(\omega)$.

Besides selfduality, there is a more general concept of symmetry, which is used even outside algebra; it is based on automorphisms. For a lattice $L$, let $\operatorname{Aut}(L)$ denote the automorphism group of $L$. We call a subset (or a sublattice) $S$ of $L$ symmetric if $\pi(S)=S$ for every $\pi \in \operatorname{Aut}(F)$. Also, an element $u \in L$ is a symmetric element of $L$ if $\{u\}$ is a symmetric subset of $L$. Note that there is no symmetric element in $\operatorname{FL}(\omega)$. If $S$ contains only symmetric elements of $L$, then $S$ is element-wise symmetric in $L$. Our key concept is the following; Theorem 1.2 and Remark 2.1 will explain why.

Definition 1.1. A lattice embedding $\varphi: L \rightarrow F$ of a lattice $L$ into a free lattice $F$ is totally symmetric if its range $\varphi(L)=\{\varphi(u): u \in L\}$ is a selfdually positioned and element-wise symmetric sublattice of $F$.

To expand our notation for all cardinal numbers $\kappa \geq 3$, we denote by $\mathrm{FL}(\kappa)$ the free lattice with $\kappa$ many free generators. If $\kappa=n$ is a natural number, then we often write $\operatorname{FL}(n)$. Following the tradition, we often denote $\mathrm{FL}\left(\aleph_{0}\right)$ by $\mathrm{FL}(\omega)$. In order to avoid ambiguity about natural numbers, we adhere to the notations $\mathbb{N}^{+}:=\{1,2,3, \ldots\}$ and $\mathbb{N}_{0}:=\{0\} \cup \mathbb{N}^{+}$. The elements of $\mathbb{N}_{0}$ are also cardinals; namely, the finite cardinal numbers. Our main result is the following. 


\section{Theorem 1.2.}

(A) Assuming that $3 \leq \kappa$ and $3 \leq \lambda$ are cardinal numbers, there exists $a$ totally symmetric embedding $\mathrm{FL}(\kappa) \rightarrow \mathrm{FL}(\lambda)$ if and only if $\lambda \in \mathbb{N}^{+}$is a natural number and $\kappa \in\left\{2 k: k \in \mathbb{N}^{+}\right\} \cup\left\{\aleph_{0}\right\}$.

(B) In particular, there exists a totally symmetric embedding of $\mathrm{FL}(\omega)$ into $\mathrm{FL}(3)$.

For later reference, we mention the following corollary even if it trivially follows from Theorem 1.2.

Corollary 1.3. There exists a totally symmetric embedding $\mathrm{FL}(4) \rightarrow \mathrm{FL}(3)$.

In addition to Theorem 1.2 on total symmetry, we have some progress in studying selfdually positioned free sublattices, which is stated as follows.

Theorem 1.4. Assuming that $3 \leq \kappa$ and $3 \leq \lambda$ are cardinal numbers, $\operatorname{FL}(\lambda)$ has a selfdually positioned sublattice isomorphic to $\mathrm{FL}(\kappa)$ if and only if the inequality $\max \left\{\kappa, \aleph_{0}\right\} \leq \max \left\{\lambda, \aleph_{0}\right\}$ holds.

This theorem is stronger than (1.2), the main result of Czédli [2]. Implicitly, the particular case of Theorem 1.4 where $\lambda=3$ and $\kappa$ belongs to $\left\{\aleph_{0}\right\} \cup\left\{2 k: k \in \mathbb{N}^{+}\right\}$is also included in [2].

\section{Prerequisites}

The reader is expected to have some basic familiarity only with the rudiments of lattice theory. That is, only some preliminary sections of the monographs, say, Grätzer [12] or Nation [16] are assumed. The results on free lattices that we need from the literature, mainly from Freese, Ježek, and Nation [9], are known for most lattice theorists and will be quoted with sufficient details since the paper is intended to be self-contained. In Section 5, we quote some recent achievements from [2]; when reading this section, the reader does not have to but may want to look into Czédli [2] to verify how we quote from it.

\section{Main ideas of the paper}

In this subsection, we deal mainly with the totally symmetric embeddability of $\operatorname{FL}(\omega)$ into $\mathrm{FL}(3)$, that is, with Part (B) of Theorem 1.2; the rest of the results are derived from or proved like Theorem 1.2(B), or they are easier. This subsection references several lemmas that will be formulated only in later sections of the paper; the reader may want to postpone understanding some details in these lemmas.

First, we define symmetric elements $m_{1}<\cdots<m_{4}$ in $\operatorname{FL}(3)$, see (3.2), and $\overline{m_{i}}$ will be the dual of $m_{i}$ for $i \in\{1, \ldots, 4\}$. With some computation based on Whitman's condition, we can prove that

(plan1) $P=\left\{m_{1}, \ldots, m_{4}\right\} \cup\left\{\overline{m_{1}}, \ldots, \overline{m_{4}}\right\}$ is the cardinal sum of two 4-element chains; see Figure 1 and Lemmas 4.7 and 4.9 for an illustration and for proofs, respectively, and

(plan2) we prove some properties of $P$ implying that the sublattice $[P]_{\mathrm{FL}(3)}$ generated by $P$ in $\mathrm{FL}(3)$ is isomorphic to the completely free lattice $\mathrm{CF}(P ; \leq)$ generated by the ordered set $(P ; \leq)$; see Corollary 4.10 . 


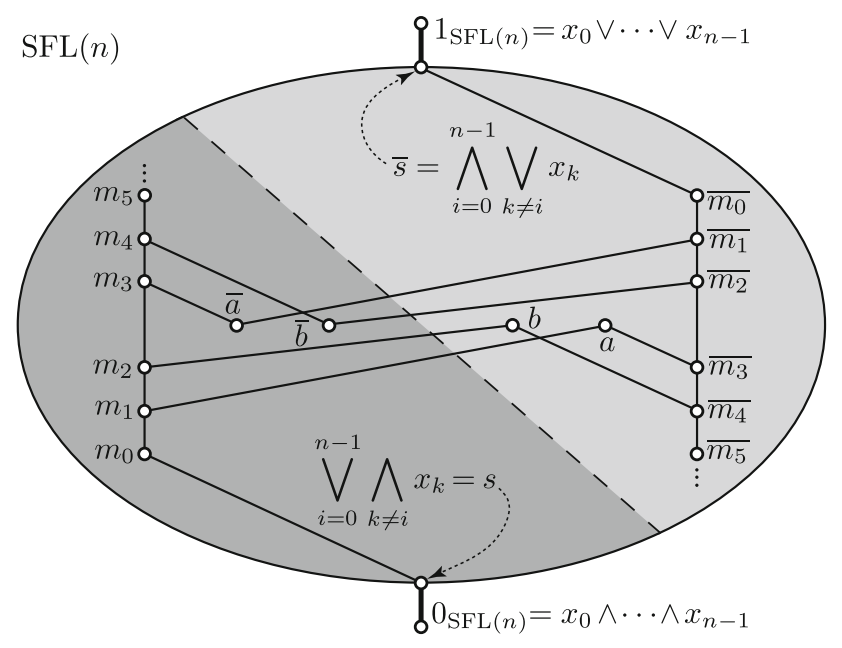

Figure 1. The lattice $\operatorname{SFL}(n)$ for $n>3$

Combining the isomorphism $[P]_{\mathrm{FL}(3)} \cong \mathrm{CF}(P ; \leq)$ with the main result of Rival and Wille [17], we could immediately obtain an element-wise symmetric sublattice $S$ of $\mathrm{FL}(3)$ such that $S \cong \mathrm{FL}(\omega)$. However, we want more. Hence, (plan3) we define $a, b \in[P]_{\mathrm{FL}(3)}$ in (3.2), see also Figure 1, such that some computation based on Whitman's condition yields that FL(4) is isomorphic to $[a, b, \bar{a}, \bar{b}]_{\mathrm{FL}(3)}$, the sublattice of $\mathrm{FL}(3)$ generated by $\{a, b, \bar{a}, \bar{b}\}$, where $\bar{a}$ and $\bar{b}$ are the duals of $a$ and $b$, respectively; see Lemma 3.1 for a more general statement. Note that the restriction of $\delta_{\mathrm{FL}(3)}$ to $[a, b, \bar{a}, \bar{b}]_{\mathrm{FL}(3)} \cong$ $\mathrm{FL}(4)$ is not the natural dual automorphism of the free lattice $[a, b, \bar{a}, \bar{b}]_{\mathrm{FL}(3)}$ since it swaps the free generators of $[a, b, \bar{a}, \bar{b}]_{\mathrm{FL}(3)}$.

At this stage, Corollary 1.3 is already proved. Next, let $\delta_{4}^{\text {sw }}$ denote the unique dual automorphism of $\mathrm{FL}(4)=\mathrm{FL}\left(y_{0}, y_{1}, y_{2}, y_{3}\right)$ for which we have that $\delta_{4}^{\text {sw }}\left(y_{0}\right)$ $=y_{1}, \delta_{4}^{\mathrm{sw}}\left(y_{1}\right)=y_{0}, \delta_{4}^{\mathrm{sw}}\left(y_{3}\right)=y_{4}, \delta_{4}^{\mathrm{sw}}\left(y_{4}\right)=y_{3}$; we call it the swapping dual automorphism of $\mathrm{FL}(4)$.

(plan4) By the "diagonal method" of Czédli [2], $\mathrm{FL}(\omega)$ is isomorphic to a sublattice of FL(4) closed with respect to $\delta_{4}^{\text {sw }}$.

Finally, a straightforward computation will show that if we combine (plan3) and (plan4), then their "swapping" features neutralize each other and we obtain a totally symmetric embedding $\mathrm{FL}(\omega) \rightarrow \mathrm{FL}(3)$, as required; a generalized form of this computation is given in the proof of Lemma 5.1.

If we embed $\mathrm{FL}(\omega)$ or $\mathrm{FL}(\kappa)$ into $\mathrm{FL}(\lambda)$, rather than into $\mathrm{FL}(3)$, then some of the above-mentioned computations, most of which can be done by a computer, become longer. Fortunately, we can often rely on the following fact, which deserves separate mentioning here: with two trivial exceptions, every symmetric element of $\mathrm{FL}(\lambda)$ is given by a near-unanimity term; see Lemma 4.4.

Let us note that the isomorphism $[P]_{\mathrm{FL}(3)} \cong \mathrm{CF}(P ; \leq)$ and the abovementioned result of Rival and Wille [17] are only motivating facts and will 
not be used in the detailed proof. Note also that this subsection will not be used in the rest of the paper; due to elaborated details and many internal references, the proofs are readable without keeping the main ideas in mind. Finally, since we also need to prove (our second) Theorem 1.4, we will prove more on $\{a, b, \bar{a}, \bar{b}\}$ than what is required by (plan3); see Lemma 3.1.

\section{Outline}

Our main results, Theorems 1.2 and 1.4, and our main ideas have already been presented; the rest of the paper is structured as follows. We add some comments and two corollaries to the main result in Section 2. These corollaries characterize the pairs $(\kappa, \lambda)$ of cardinals having the property that there is an embedding $\mathrm{FL}(\kappa) \rightarrow \mathrm{FL}(\lambda)$ with symmetric range or with selfdually positioned and symmetric range. The lion's share of our construction and (the Key) Lemma 3.1 stating that this construction works are given in Section 3. The Key Lemma is proved in Section 4. Section 5 combines the construct given in Section 3 with that given in Czédli [2]. Section 6 completes the proofs of our theorems and proves the corollaries. Finally, Section 7 describes our computer program for the word problem of free lattices; note that this program and its source file are freely available and the program proves Corollary 1.3 in less than a millisecond.

\section{Remarks and corollaries}

We will often use the convenient notation $\mathrm{FL}(\kappa)=\mathrm{FL}\left(x_{i}: i<\kappa\right)$ in order the specify the free generating set $\left\{x_{i}: i<\kappa\right\}$ of size $\kappa$; in this case, $i$ denotes an ordinal number and $i<\kappa$ is understood as $|i|<\kappa$. An element $u$ of a lattice $L$ is doubly irreducible if $L \backslash\{u\}$ is closed with respect to both joins and meets, that is, if $u$ is both join irreducible and meet irreducible. The set of doubly irreducible elements of $L$ will be denoted by $\operatorname{Irr}_{\wedge}^{\vee}(L)$. We know from Whitman [20], see also Corollary 1.9 and the first sentence of the proof of Corollary 1.12 in Freese, Ježek, and Nation [9], that

$$
\begin{aligned}
& \operatorname{Irr}_{\wedge}^{\vee}(\operatorname{FL}(\kappa))=\left\{x_{i}: i<\kappa\right\}, \text { and } \\
& \text { every element of } \operatorname{FL}(\kappa) \text { is join or meet irreducible. }
\end{aligned}
$$

Note that we consider 0 and 1 join irreducible and meet irreducible, respectively, if these elements exist. Since a dual automorphism maps join-irreducible elements to meet irreducible ones and vice versa, (2.1) and (2.2) imply that for every dual automorphism $\psi$ of $\mathrm{FL}(\kappa)$, we have that

$$
\{u: \psi(u)=u\} \subseteq \psi\left(\left\{x_{i}: i<\kappa\right\}\right)=\left\{x_{i}: i<\kappa\right\} .
$$

Remark 2.1. The concept of totally symmetric embeddings might raise the question whether we could consider even stronger embeddings whose ranges are element-wise symmetric and are in element-wise selfdual positions. We obtain from (2.3) that the answer is negative, since at most the free generators are in element-wise selfdual positions and they form an antichain. This justifies our terminology to call the embeddings in Theorem 1.2 totally symmetric. 
Remark 2.2. Assume that $3 \leq \kappa \leq \aleph_{0}$ and $3 \leq \lambda \leq \aleph_{0}$; as a comparison between the result of Whitman [20] and Theorem 1.2, note the following. It follows immediately from Whitman' result that $\mathrm{FL}(\kappa)$ is embeddable into $\operatorname{FL}(\lambda)$, because embeddability is a transitive relation and $\gamma_{1} \leq \gamma_{2}$ implies that $\operatorname{FL}\left(\gamma_{1}\right)$ is embeddable into $\mathrm{FL}\left(\gamma_{2}\right)$. However, the analogous implication fails for totally symmetric embeddability, since a symmetric element of $\operatorname{FL}\left(\gamma_{1}\right)$ is not symmetric in $\operatorname{FL}\left(\gamma_{2}\right)$ for $\gamma_{2}>\gamma_{1}$. This explains that, as opposed to Whitman's result, Theorem 1.2 contains two parameters, $\kappa$ and $\lambda$.

For a selfdual lattice $L$, let $\operatorname{DAut}(L)$ be the set of all automorphisms and dual automorphisms of $L$. As a consequence of (2.1) and (2.2), note that for $\kappa \leq \omega$,

$\operatorname{Irr}_{\wedge}^{\vee}(\mathrm{FL}(\kappa))$ is closed with respect to every $\pi \in \operatorname{DAut}(\mathrm{FL}(\kappa))$. Furthermore, each $\pi \in \operatorname{DAut}(\mathrm{FL}(\kappa))$ is determined by its restriction to $\operatorname{Irr}_{\wedge}^{\vee}(\mathrm{FL}(\kappa))$ if we know whether it is an automorphism or a dual automorphism.

With respect to composition, $\operatorname{DAut}(L)$ is a group and $\operatorname{Aut}(L)$ is a normal subgroup in it with index $[\operatorname{DAut}(L): \operatorname{Aut}(L)]=2$. Let us call a subset $S$ of $L$ a DAut-symmetric subset if $\pi(S)=S$ for all $\pi \in \operatorname{DAut}(L)$. A dually positioned and element-wise symmetric sublattice of $\mathrm{FL}(\lambda)$, like the range of a totally symmetric embedding, is clearly DAut-symmetric but not conversely. This might give some hope that a counterpart of Theorem 1.2 for embeddings with DAut-symmetric ranges would allow the case when $\kappa$ is an odd natural number. However, the following corollary of Theorem 1.2 shows that this is not so if $\kappa \neq \lambda$. This corollary as well as Corollary 2.4 will be proved in Section 6 .

Corollary 2.3. Assuming that $3 \leq \kappa$ and $3 \leq \lambda$ are cardinal numbers, the following two conditions are equivalent.

(i) There exists an embedding $\mathrm{FL}(\kappa) \rightarrow \mathrm{FL}(\lambda)$ with DAut-symmetric range.

(ii) Either $\kappa=\lambda$, or we have that $\lambda \in \mathbb{N}^{+}$and $\kappa \in\left\{2 k: k \in \mathbb{N}^{+}\right\} \cup\left\{\aleph_{0}\right\}$.

The situation is different if we deal with embeddings whose ranges are symmetric with respect only to $\operatorname{Aut} \mathrm{FL}(\lambda)$.

Corollary 2.4. Assuming that $3 \leq \kappa$ and $3 \leq \lambda$ are cardinal numbers, there exists an embedding $\mathrm{FL}(\kappa) \rightarrow \mathrm{FL}(\lambda)$ with symmetric range if and only if either $\lambda \in \mathbb{N}^{+}$and $\kappa \leq \aleph_{0}$, or $\kappa=\lambda \geq \aleph_{0}$.

Since our concepts are based on the automorphisms of $\mathrm{FL}(n)$, where $n:=\lambda$ is a positive integer, let us have a look at what these automorphisms and the elements of $\operatorname{DAut}(\mathrm{FL}(n))$ are. Let $\operatorname{Sym}_{n}=\operatorname{Sym}(\{0,1, \ldots, n-1\})$ denote the group of all permutations of $\{0,1, \ldots, n-1\}$ with respect to composition, and let $C_{2}=\{1,-1\}$ be the cyclic group of order 2 , considered a subgroup of the group $(\mathbb{R} \backslash\{0\} ; \cdot)$ of nonzero real numbers with respect to multiplication. Using that $\mathrm{FL}(n)=\mathrm{FL}\left(x_{i}: i<n\right)$ is freely generated by the set $\left\{x_{i}: i<n\right\}$, the following remark is straightforward and its proof is omitted.

Remark 2.5. For $n \in \mathbb{N}^{+}$and the free lattice $\operatorname{FL}(n)=\operatorname{FL}\left(\left\{x_{i}: i<n\right\}\right)$, the groups $\operatorname{Aut}(\mathrm{FL}(n))$ and $\operatorname{DAut}(\mathrm{FL}(n))$ are isomorphic to $\operatorname{Sym}_{n}$ and $\operatorname{Sym}_{n} \times \mathrm{C}_{2}$, 
respectively. More specifically, for $\sigma \in \operatorname{Sym}_{n}$, let $\sigma^{\text {aut }}: \operatorname{FL}(n) \rightarrow \operatorname{FL}(n)$ and $\overline{\sigma^{\text {aut }}}: \mathrm{FL}(n) \rightarrow \mathrm{FL}(n)$ be the maps defined by

$$
\begin{aligned}
& \sigma^{\text {aut }}\left(t\left(x_{0}, \ldots, x_{n-1}\right)\right):=t\left(x_{\sigma(0)}, \ldots, x_{\sigma(n-1)}\right) \text { and } \\
& \overline{\sigma^{\text {aut }}}\left(t\left(x_{0}, \ldots, x_{n-1}\right)\right):=\delta_{\mathrm{FL}(n)}\left(t\left(x_{\sigma(0)}, \ldots, x_{\sigma(n-1)}\right)\right),
\end{aligned}
$$

respectively, where $t$ denotes an $n$-ary lattice term and $\delta=\delta_{\mathrm{FL}(n)}$ has been defined in (1.1). Then the maps

$$
\begin{aligned}
& \operatorname{Sym}_{n} \rightarrow \operatorname{Aut}(\mathrm{FL}(n)), \text { defined by } \sigma \mapsto \sigma^{\text {aut }}, \text { and } \\
& \operatorname{Sym}_{n} \times \mathrm{C}_{2} \rightarrow \operatorname{DAut}(\mathrm{FL}(n)), \text { defined by }(\sigma, k) \mapsto \begin{cases}\overline{\sigma^{\text {aut }},} & \text { if } k=-1, \\
\sigma^{\text {aut }}, & \text { if } k=1\end{cases}
\end{aligned}
$$

are group isomorphisms. (In particular, they are well defined maps.)

\section{Construction and the Key Lemma}

\subsection{Notation}

The elements of a free lattice $\mathrm{FL}(\kappa)=\mathrm{FL}\left(x_{i}: i<\kappa\right)$ will be represented by lattice terms over the set $\left\{x_{i}: i<\kappa\right\}$ of variables. Although there are many terms representing the same element of $\mathrm{FL}(\kappa)$, it will not cause any confusion that

we often treat and call these terms as elements of the free lattice;

see pages 10-11 in Freese, Ježek and Nation [9] for a more rigorous setting. The dual of a term $t$ will always be denoted by $\bar{t}$; the overline reminds us that dualizing at visual level means to reflect Hasse diagrams across horizontal axes. The Symmetric part of the Free Lattice of $\mathrm{FL}(\kappa)$ will be denoted as follows; capitalization explains the acronym:

$$
\operatorname{SFL}(\kappa):=\{u \in \mathrm{FL}(\kappa): \pi(u)=u \text { for all } \pi \in \operatorname{Aut} \mathrm{FL}(\kappa)\} .
$$

Clearly, $\operatorname{SFL}(\kappa)$ is a sublattice of $\mathrm{FL}(\kappa)$; this fact will often be used implicitly.

\subsection{Constructing some important terms}

In this subsection, we give a construction for the particular case $(\kappa, \lambda)=(4, n)$ of Theorem 1.2(A). Let us agree to the following conventions. The set

$$
\{0,1, \ldots, n-1\} \text { will also be denoted by }[n) \text {. }
$$

The inequality $i<n$ is equivalent to $i \in[n)$. Whenever $x_{i}, x_{j}$, etc. refer to a free generator of $\mathrm{FL}(n)=\mathrm{FL}\left(x_{0}, \ldots, x_{n-1}\right)$, then $i, j, \ldots$ will automatically belong to $[n)$; this convention will often save us from indicating, say, that $i<n$ or $i, j \in[n)$ below the $\bigvee$ and $\bigwedge$ operation signs. Also, we frequently abbreviate the conjunction of $i \in[n)$ and $j \in[n) \backslash\{i\}$ by the short form $i \neq j$, and selfexplanatory similar other abbreviations may also occur. For the rest of this section, let $3 \leq n=\lambda \in \mathbb{N}^{+}$and

$$
\mathrm{FL}(\lambda)=\mathrm{FL}(n)=\mathrm{FL}\left(x_{0}, \ldots, x_{n-1}\right)=\mathrm{FL}\left(x_{i}: i<n\right) .
$$


By induction on $j$, we define the following $n$-ary lattice terms over the set $\left\{x_{i}: i<n\right\}$ of variables; according to (3.1), they will also be considered elements of $\operatorname{FL}(n)$. Namely, we let

$$
\begin{aligned}
p_{0}^{(i)} & :=x_{i} \text { for } i \in\{0,1, \ldots, n-1\}=[n), \\
p_{j}^{(i)} & :=x_{i} \vee \bigvee_{\substack{i_{1}<i_{2} \\
i_{1}, i_{2} \in[n) \backslash\{i\}}}\left(p_{j-1}^{\left(i_{1}\right)} \wedge p_{j-1}^{\left(i_{2}\right)}\right) \text { for } i \in[n) \text { and } j \in \mathbb{N}^{+}, \\
m_{j} & :=\bigvee_{i_{1}<i_{2}, i_{1}, i_{2} \in[n)}\left(p_{j}^{\left(i_{1}\right)} \wedge p_{j}^{\left(i_{2}\right)}\right) \text { for } j \in \mathbb{N}_{0}, \\
a & :=m_{1} \vee \overline{m_{3}}, \text { and } b:=m_{2} \vee \overline{m_{4}} .
\end{aligned}
$$

For later reference, we note that the set

$$
\{a, \bar{a}, b, \bar{b}\}
$$

will play an important role in the paper. We say that a subset $X$ of a lattice freely generates if the sublattice $S$ generated by $X$ is a free lattice with $X$ as the set of free generators. Next, we formulate our Key Lemma, which is stronger than asserting that the set in (3.3) freely generates. The proof of the Key Lemma will be postponed to Section 4.

Lemma 3.1 (Key Lemma). If $3 \leq n \in \mathbb{N}^{+}$, then the elements $m_{j}$ and $\overline{m_{j}}$ for $j \in \mathbb{N}_{0}, a, b, \bar{a}$, and $\bar{b}$ all belong to $\operatorname{SFL}(n)$. Furthermore, $\left\{a, \bar{a}, b, \bar{b}, x_{0}\right\}$ is a five-element subset of $\operatorname{SFL}(n)$ that freely generates.

For later reference, based on Remark 2.5, note the following trivial lemma.

Lemma 3.2. For every $i \in[n), j \in \mathbb{N}_{0}$ and $\sigma \in \operatorname{Sym}([n))$, we have that $\sigma^{\text {aut }}\left(p_{j}^{(i)}\right)=p_{j}^{(\sigma(i))}$ and $\sigma^{\text {aut }}\left(m_{j}\right)=m_{j}$.

Proof of Lemma 3.2. The first equality above follows from the fact that in (3.2), each stipulation of the form $i_{1}<i_{2}$ can be replaced by $i_{1} \neq i_{2}$ by idempotence. The second equality follows from the first one.

\section{The proof of the Key Lemma}

From the theory of free lattices, we only use three basic facts, which we recall below as lemmas; all of them can be found in Freese, Ježek and Nation [9]. An element $u$ of a lattice $L$ is join prime if for all $k \in \mathbb{N}^{+}$and $x_{0}, \ldots, x_{k-1} \in L$, the inequality $u \leq x_{0} \vee \cdots \vee x_{k-1}$ implies that $u \leq x_{i}$ for some $i \in[k)$. Meet prime elements are defined dually. An element is doubly prime if it is join prime and meet prime.

Lemma 4.1 (Whitman [20]; Freese, Ježek and Nation [9, Corollary 1.5]). In every free lattice $\mathrm{FL}(X)$, the free generators are doubly prime elements. 
The following statement says that free lattices satisfy Whitman's condition (W), see Whitman [20].

Lemma 4.2 (Whitman [20]; Freese, Ježek and Nation [9, Theorem 1.8]). For arbitrary elements $u_{1}, \ldots, u_{r}, v_{1}, \ldots, v_{s}$ of a free lattice $\mathrm{FL}(X)$,

(W) The inequality $u=u_{1} \wedge \cdots \wedge u_{r} \leq v_{1} \vee \cdots \vee v_{s}=v$ implies that either $u_{i} \leq v$ for some subscript $i$, or $u \leq v_{j}$ for some $j$.

Next, we describe whether a subset of a free lattice generates freely or not.

Lemma 4.3 (Whitman [20]; Freese, Ježek and Nation [9, Corollary 1.13]). A nonempty subset $Y$ of $\mathrm{FL}(X)$ generates freely if and only if for all $h \in Y$ and all finite subsets $Z \subseteq Y$, the following condition and its dual hold.

$$
h \notin Z \text { implies } h \notin \bigvee_{z \in Z} z \text {. }
$$

For the rest of this section, assume that $3 \leq n \in \mathbb{N}^{+}$. A (lattice) term $t$ is called a near-unanimity lattice term or, shortly, an NU-term if it satisfies

$$
t(y, x, \ldots, x)=t(x, y, x, \ldots, x)=\cdots=t(x, \ldots, x, y)=x .
$$

Since the lattice operations are idempotent, it is obvious that

the join and the meet of two $n$-ary NU-terms are NU-terms.

If $t_{1}$ and $t_{2}$ are $n$-ary lattice terms such that $t_{1}=t_{2}$ in $\operatorname{FL}(n)$, see (3.1), then $t_{1} \in \operatorname{SFL}(n)$ iff $t_{2} \in \operatorname{SFL}(n)$. Also, for $t_{1}=t_{2} \in \mathrm{FL}(n), t_{1}$ is an NU-term iff so is $t_{2}$. So convention (3.1) still applies.

Lemma 4.4. If $t \in \operatorname{SFL}(n)=\operatorname{SFL}\left(x_{0}, \ldots, x_{n-1}\right)$ such that neither of the equalities $t\left(x_{0}, \ldots, x_{n-1}\right)=\bigwedge_{i \in[n)} x_{i}$ and $t\left(x_{0}, \ldots, x_{n-1}\right)=\bigvee_{i \in[n)} x_{i}$ holds in $\mathrm{FL}(n)$, then $t$ is a near-unanimity term.

Proof. It is straightforward to see that

$$
\begin{aligned}
& \text { if } g \in \operatorname{SFL}(n) \text { and } x_{i} \leq g \text { in } \operatorname{FL}(n) \text { for some } i \in[n) \text {, } \\
& \text { then } g=1_{\operatorname{SFL}(n)}=x_{0} \vee \cdots \vee x_{n-1} \text {, and dually. }
\end{aligned}
$$

Next, assume that $t$ satisfies the assumptions of the lemma. As a binary lattice term, $t(x, \ldots, x, y)$ equals one of $x, y, x \wedge y$, and $x \vee y$ in $\operatorname{FL}(x, y)$. If we had that, in $\mathrm{FL}(x, y), t(x, \ldots, x, y)=x \wedge y$, then

$$
\begin{aligned}
t\left(x_{0}, \ldots, x_{n-1}\right) & \leq t\left(x_{0} \vee \cdots \vee x_{n-2}, \ldots, x_{0} \vee \cdots \vee x_{n-2}, x_{n-1}\right) \\
& \leq\left(x_{0} \vee \cdots \vee x_{n-2}\right) \wedge x_{n-1} \leq x_{n-1}
\end{aligned}
$$

together with (4.2) would yield that $t=0_{\mathrm{SFL}(n)}=x_{0} \wedge \cdots \wedge x_{n-1}$, a contradiction. Hence, $t(x, \ldots, x, y)$ is distinct from $x \wedge y$, and it is distinct also from $x \vee y$ by duality. The case $t(x, x, \ldots, x, y)=y$ is impossible, because it would imply that $1=t(0, \ldots, 0,1) \leq t(0,1, \ldots, 1)=t(1, \ldots, 1,0)=0$ holds in the two-element lattice $\mathbf{2}$, which is a contradiction. Hence, $t(x, x, \ldots, x, y)=x$, which means that $t$ is an NU-term since it is symmetric. 
Lemma 4.5. There is exactly one atom in $\operatorname{SFL}(n)$, and it is

$$
s:=\bigvee_{i \in[n)} \bigwedge_{i^{\prime} \in[n) \backslash\{i\}} x_{i^{\prime}} .
$$

The only coatom of $\operatorname{SFL}(n)$ is $\bar{s}$. Except from its bottom $0_{\mathrm{SFL}(n)}=\bigwedge_{i<n} x_{i}$ and top $1_{\mathrm{SFL}(n)}=\bigvee_{i<n} x_{i}$, every element of $\operatorname{SFL}(n)$ is in the interval $[s, \bar{s}]$.

The statement of this lemma for $3<n \in \mathbb{N}^{+}$is illustrated by Figure 1, where only the two thick edges stand for coverings in $\operatorname{SFL}(n)$; the thin lines indicate comparabilities that need not be coverings. (These comparabilities will be proved later; see Lemma 4.9.) The reflection across the symmetry center point, which is not indicated in the figure, represents the restriction of $\delta_{\mathrm{FL}(n)}$ to $\operatorname{SFL}(n)$. We could obtain a similar figure for $n=3$ by removing the vertices $m_{0}$ and $\overline{m_{0}}$ and decreasing the subscripts of the remaining vertices by 1 ; see Lemma 4.9. Note that, as opposed to $\operatorname{SFL}(n)$, the free lattice $\operatorname{FL}(n)$ has exactly $n$ atoms; for more details, the reader is referred to the discussion of the bottom of FL $(n)$ in Freese, Ježek, and Nation [9, Section III.7].

Proof. By Lemma 4.4 and the duality principle, we need to show only that $t \geq s$ holds for every near-unanimity term $t \in \operatorname{SFL}(n)$. This follows from the fact that for each $i \in[n)$,

$$
\begin{aligned}
& t\left(x_{0}, \ldots, x_{i-1}, x_{i}, x_{i+1}, \ldots x_{n-1}\right) \\
& \geq t\left(\bigwedge_{i^{\prime} \neq i} x_{i^{\prime}}, \ldots, \bigwedge_{i^{\prime} \neq i} x_{i^{\prime}}, x_{i}, \bigwedge_{i^{\prime} \neq i} x_{i^{\prime}}, \ldots, \bigwedge_{i^{\prime} \neq i} x_{i^{\prime}}\right)=\bigwedge_{i^{\prime} \neq i} x_{i^{\prime}} .
\end{aligned}
$$

In order to get some preliminary insight into $\operatorname{SFL}(n)$, note the following. As usual, $\mathrm{M}_{n}$ denotes $(n+2)$-element lattice given in Figure 2. For a permutation $\pi:[n) \rightarrow[n)$, let $\pi_{X}$ and $\pi_{A}$ denote the permutation of $\left\{x_{i}: i<n\right\}$ and that of $\left\{a_{i}: i<n\right\}$ defined by $\pi_{X}\left(x_{i}\right)=x_{\pi(i)}$ and $\pi_{A}\left(a_{i}\right)=a_{\pi(i)}$ for all $i<n$, respectively. These permutations uniquely extend to automorphisms $\pi_{X}^{\text {aut }} \in \operatorname{Aut}(\operatorname{SFL}(n))$ and $\pi_{A}^{\text {aut }} \in \operatorname{Aut}\left(\mathrm{M}_{n}\right)$, respectively. Consider the natural homomorphism

$$
\eta: \mathrm{FL}(n) \rightarrow \mathrm{M}_{n} \text { defined by } \eta\left(t\left(x_{0}, \ldots, x_{n-1}\right)\right)=t\left(a_{0}, \ldots, a_{n-1}\right) .
$$

Clearly, $\pi_{A}^{\text {aut }} \circ \eta=\eta \circ \pi_{X}^{\text {aut }}$. This implies easily that the $\eta$-image of a symmetric element of $\mathrm{FL}(n)$ is a symmetric element of $\mathrm{M}_{n}$. But the symmetric elements of $\mathrm{M}_{n}$ form 2 (as a sublattice), and we obtain that $\left.\eta\right\rceil_{\operatorname{SFL}(n)}: \operatorname{SFL}(n) \rightarrow \mathbf{2}$ is a surjective homomorphism. So the kernel of $\eta\rceil_{\mathrm{SFL}(n)}$ cuts $\operatorname{SFL}(n)$ into a prime ideal, the dark-grey lower part together with the bottom element in Figure 1,

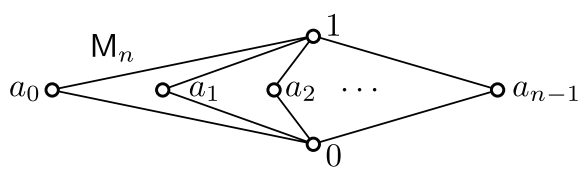

Figure 2. The lattice $\mathrm{M}_{n}$ 
and its complementary prime filter, the light-grey upper part together with the top in the figure. Besides that their shades are distinct in the figure, the two parts are separated by a dashed line.

Lemma 4.6. For every $i<n$, the sequence $\left\{p_{j}^{(i)}: j \in \mathbb{N}_{0}\right\}$ is strictly increasing, that is, $p_{0}^{(i)}<p_{1}^{(i)}<p_{2}^{(i)}<\cdots$ in $\mathrm{FL}(n)$.

Proof. First, we show by induction on $j$ that

$$
p_{j}^{(r)} \wedge p_{j}^{(s)} \not \leq x_{k} \text { for all } j \in \mathbb{N}^{+} \text {and } k, r, s \in[n) .
$$

Suppose, for a contradiction, that (4.4) fails, and let $j \in \mathbb{N}^{+}$be the smallest number violating it. Pick $k, r, s \in[n)$ such that $p_{j}^{(r)} \wedge p_{j}^{(s)} \leq x_{k}$. Since $x_{k}$ is meet prime by Lemma 4.1, we can assume that $p_{j}^{(r)} \leq x_{k}$. By (3.2), $x_{r} \leq x_{k}$, whence $r=k$ and we have that $p_{j}^{(k)} \leq x_{k}$. Pick $r^{\prime}<s^{\prime}$ in $[n) \backslash\{k\}$; this is possible since $n \geq 3$. Since $p_{j}^{(k)} \leq x_{k}$, (3.2) gives that $p_{j-1}^{\left(r^{\prime}\right)} \wedge p_{j-1}^{\left(s^{\prime}\right)} \leq x_{k}$. Since this inequality does not violate (4.4) by the choice of $j$, it follows that $j-1 \notin \mathbb{N}^{+}$, whence $j=1$. Then (3.2) turns $p_{j-1}^{\left(r^{\prime}\right)} \wedge p_{j-1}^{\left(s^{\prime}\right)} \leq x_{k}$ into $x_{r^{\prime}} \wedge x_{s^{\prime}} \leq x_{k}$, which is a contradiction since $k \notin\left\{r^{\prime}, s^{\prime}\right\}$. This proves (4.4).

Next, based on (3.2), a trivial induction on $j$ shows that

for all $j \in \mathbb{N}_{0}$ and $i \in[n), p_{j}^{(i)}\left(a_{0}, \ldots, a_{n-1}\right)=a_{i}$ holds in $\mathrm{M}_{n}$.

This implies that, for any $j, j^{\prime} \in \mathbb{N}_{0}$ and $i, i^{\prime} \in[n)$,

if $i \neq i^{\prime}$, then the terms $p_{j}^{(i)}$ and $p_{j^{\prime}}^{\left(i^{\prime}\right)}$ are incomparable in $\operatorname{FL}(n)$.

A straightforward induction yields that the sequence is increasing, that is,

$$
p_{0}^{(i)} \leq p_{1}^{(i)} \leq p_{2}^{(i)} \leq \cdots \text { holds in } \operatorname{FL}(n) .
$$

Armed with the preparations above, it suffices to prove the strict inequalities in the lemma only for $i=0$, since then the case $i>0$ will follow by symmetry. For the sake of contradiction, suppose that there exists a $j \in \mathbb{N}_{0}$ such that

$$
p_{j+1}^{(0)} \stackrel{(3.2)}{=} x_{0} \vee \bigvee_{i_{1}<i_{2},} \bigvee_{i_{1}, i_{2} \in[n) \backslash\{0\}}\left(p_{j}^{\left(i_{1}\right)} \wedge p_{j}^{\left(i_{2}\right)}\right) \leq p_{j}^{(0)}
$$

Let $j$ be minimal with this property. By symmetry, the superscript 0 does not play a distinguished role here. That is, until the end of the proof,

$$
\text { for each } i \in[n), j \in \mathbb{N}_{0} \text { is minimal such that } p_{j+1}^{(i)} \leq p_{j}^{(i)} \text {. }
$$

We are going to derive a contradiction from (4.8) by infinite descent. Since $p_{1}^{(0)}=x_{0} \vee\left(x_{1} \wedge x_{2}\right) \vee \cdots \leq x_{0}=p_{0}^{(0)}$ would lead to $x_{1} \wedge x_{2} \leq x_{0}$, which 
fails even in $\mathbf{2}$, we obtain that $j>0$. So, as the first step of the descent, we conclude that $j-1 \in \mathbb{N}_{0}$. Thus, (4.8) and $j-1 \in \mathbb{N}_{0}$ imply that

$$
p_{j}^{(1)} \wedge p_{j}^{(2)} \leq p_{j}^{(0)} \stackrel{(3.2)}{=} x_{0} \vee \underset{i_{1}<i_{2}, i_{1}, i_{2} \in[n) \backslash\{0\}}{\bigvee}\left(p_{j-1}^{\left(i_{1}\right)} \wedge p_{j-1}^{\left(i_{2}\right)}\right) .
$$

By (4.6), this inequality would fail if one of the two meetands on the left was omitted. Hence, $(\mathrm{W})$ and (4.4) yield that $p_{j}^{(1)} \wedge p_{j}^{(2)} \leq p_{j-1}^{\left(u_{1}\right)} \wedge p_{j-1}^{\left(v_{1}\right)}$ for some $v_{1}, u_{1} \in[n) \backslash\{0\}$. In particular, $p_{j}^{(1)} \wedge p_{j}^{(2)} \leq p_{j-1}^{\left(u_{1}\right)}$. We formulate this inequality together with $j-1 \in \mathbb{N}_{0}$ also in the following way: the condition

$$
j-m \in \mathbb{N}_{0} \text { and there is a } u_{m} \in[n) \text { such that } p_{j}^{(1)} \wedge p_{j}^{(2)} \leq p_{j-m}^{\left(u_{m}\right)}
$$

holds for $m=1$. In order to continue the descent in infinitely many steps, we assert the following.

$$
\begin{aligned}
& \text { Assume that } p_{j-1}^{(1)}<p_{j}^{(1)} \text {. Then, for every } m \in \mathbb{N}^{+} \text {, if } \\
& \text { (4.11) holds for } m \text {, then it holds also for } m+1 \text {. }
\end{aligned}
$$

(The first sentence in (4.12) is included for later reference.) In order to prove (4.12), assume (4.11) for $m$. The case $j-m=0$ is ruled out by (4.4), whence $j-(m+1) \in \mathbb{N}_{0}$. Hence, the inequality in (4.11) gives that

$$
p_{j}^{(1)} \wedge p_{j}^{(2)} \leq p_{j-m}^{\left(u_{m}\right)} \stackrel{(3.2)}{=} x_{u_{m}} \vee \bigvee_{\substack{i_{1}<i_{2} \\ i_{1}, i_{2} \in[n) \backslash\left\{u_{m}\right\}}}\left(p_{j-(m+1)}^{\left(i_{1}\right)} \wedge p_{j-(m+1)}^{\left(i_{2}\right)}\right) .
$$

As before, we are going to apply (W) to (4.13); however, the argument for the meetands on the left is a bit longer. If $u_{m} \notin\{1,2\}$, then we obtain from (4.6) that none of the meetands on the left of (4.13) can be omitted from the inequality. If $u_{m}=1$, then $p_{j}^{(1)}$ still cannot be omitted by (4.6), but we need the same fact for the other meetand, $p_{j}^{(2)}$. Observe that if $p_{j}^{(2)}$ was omitted and $u_{m}$ equaled 1 , then we would have by (4.7) that $p_{j}^{(1)} \leq p_{j-m}^{(1)} \leq p_{j-1}^{(1)}$, contradicting the first sentence of (4.12). So none of the two meetands in question can be omitted if $u_{m}=1$, and the same is true for $u_{m}=2$ since 1 and 2 play symmetric roles. This shows that no matter what is $u_{m}$, none of the two meetands on the left of (4.13) can be omitted. Therefore, (4.13), (W) and (4.4) imply that $p_{j}^{(1)} \wedge p_{j}^{(2)} \leq p_{j-(m+1)}^{\left(u_{m+1}\right)} \wedge p_{j-(m+1)}^{\left(v_{m+1}\right)}$ for some $u_{m+1}, v_{m+1} \in[n) \backslash\left\{u_{m}\right\}$. In particular, $p_{j}^{(1)} \wedge p_{j}^{(2)} \leq p_{j-(m+1)}^{\left(u_{m+1}\right)}$. Thus, we conclude that (4.11) holds for $m+1$, completing the proof of (4.12).

Finally, (4.7) and (4.9) yield the validity of the first sentence of (4.12). Consequently, it follows from (4.12) that (4.11) holds for all $m \in \mathbb{N}^{+}$, which contradicts the finiteness of $j \in \mathbb{N}_{0}$ and completes the proof of Lemma 4.6.

The following lemma is visualized by a part of Figure 1.

Lemma 4.7. The sequence $\left\{m_{j}: j \in \mathbb{N}_{0}\right\}$ is strictly increasing, that $i s$, we have that $m_{0}<m_{1}<m_{2}<\cdots$ in $\mathrm{FL}(n)$. Also, $\left\{\overline{m_{j}}: j \in \mathbb{N}_{0}\right\}$ is strictly decreasing. 
Proof. It suffices to deal only with $\left\{m_{j}: j \in \mathbb{N}_{0}\right\}$. The sequence in question is increasing by its definition, see (3.2), and Lemma 4.6. For the sake of contradiction, suppose the sequence is not strictly increasing, so that $m_{j} \leq m_{j-1}$ holds for some $j \in \mathbb{N}^{+}$. Then, since all joinands of $m_{j}$ are less than or equal to $m_{j-1}$, we have that, in particular,

$$
p_{j}^{(1)} \wedge p_{j}^{(2)} \leq m_{j}=\bigvee_{i_{1}<i_{2}, i_{1}, i_{2} \in[n)}\left(p_{j-1}^{\left(i_{1}\right)} \wedge p_{j-1}^{\left(i_{2}\right)}\right) .
$$

It follows from (4.5) that, with $\vec{a}=\left(a_{0}, \ldots, a_{n-1}\right) \in \mathrm{M}_{n} \times \cdots \times \mathrm{M}_{n}$, we have that $m_{j}(\vec{a})=0$ while $p_{j}^{(1)}(\vec{a})=a_{1}$ and $p_{j}^{(2)}(\vec{a})=a_{2}$. Hence, neither of the meetands on the left of (4.14) can be omitted without breaking the inequality. Thus, applying (W) to (4.14), it follows that $p_{j}^{(1)} \wedge p_{j}^{(2)} \leq p_{j-1}^{\left(u_{1}\right)} \wedge p_{j-1}^{\left(v_{1}\right)}$ for some $u_{1}, v_{1} \in[m)$. This yields that $p_{j}^{(1)} \wedge p_{j}^{(2)} \leq p_{j-1}^{\left(u_{1}\right)}$. Therefore, (4.11) holds for $m=1$. By Lemma 4.6, so does the assumption in the first sentence of (4.12). Consequently, it follows from (4.12) that (4.11) holds for all $m \in \mathbb{N}^{+}$, which is a contradiction since $j-m \notin \mathbb{N}_{0}$ for $m>j$.

The following lemma states something on $\operatorname{SFL}(n)$, not on $\operatorname{FL}(n)$.

Lemma 4.8. For all $j \in \mathbb{N}_{0}, m_{j}$ and $\overline{m_{j}}$ are doubly prime elements of $\operatorname{SFL}(n)$.

Proof. By duality, it suffices to deal only with $m_{j}$. In order to show that $m_{j}$ is join prime, assume that $m_{j} \leq h_{1} \vee h_{2}$ where $h_{1}, h_{2} \in \operatorname{SFL}(n)$. Remember that the containment here means that $h_{1}$ and $h_{2}$ are fixed points of every automorphism of $\mathrm{FL}(n)$. We have to show that $m_{j} \leq h_{i}$ for some $i \in\{1,2\}$. There are two cases to consider. First, assume that

$$
\text { there exists an } i \in\{1,2\} \text { such that } p_{j}^{(0)} \wedge p_{j}^{(1)} \leq h_{i} \text {. }
$$

In this case, for each $\left(u_{1}, u_{2}\right) \in[n) \times[n)$ with $u_{1}<u_{2}$, pick a permutation $\sigma \in$ $\operatorname{Sym}([n))$ such that $\sigma(0)=u_{1}$ and $\sigma(1)=u_{2}$. By Remark 2.5 and Lemma 3.2,

$$
\begin{aligned}
p_{j}^{\left(u_{1}\right)} \wedge p_{j}^{\left(u_{2}\right)} & =p_{j}^{(\sigma(0))} \wedge p_{j}^{(\sigma(1))} \\
& =\sigma^{\text {aut }}\left(p_{j}^{(0)} \wedge p_{j}^{(1)}\right) \leq \sigma^{\text {aut }}\left(h_{i}\right)=h_{i} .
\end{aligned}
$$

Forming the join of these inequalities for all meaningful pairs $\left(u_{1}, u_{2}\right)$, we obtain that $m_{j} \leq h_{i}$, as required.

Second, assume that (4.15) fails. Then (W) applied to the (side terms of the) inequality $p_{j}^{(0)} \wedge p_{j}^{(1)} \leq m_{j} \leq h_{1} \vee h_{2}$ gives that $p_{j}^{(0)} \leq h_{1} \vee h_{2}$ or $p_{j}^{(1)} \leq h_{1} \vee h_{2}$; we can assume that $p_{j}^{(0)} \leq h_{1} \vee h_{2}$. By (3.2) and Lemma 4.6, we have that $x_{0} \leq h_{1} \vee h_{2}$. Since $x_{0}$ is join prime by Lemma $4.1, x_{0} \leq h_{i}$ holds for some $i \in\{1,2\}$. Applying (4.2), we obtain that $h_{i}=1_{\mathrm{SFL}(n)}$. Hence, $m_{j} \leq 1_{\mathrm{SFL}(n)}=h_{i}$, as required. Now that both the validity and the failure of (4.15) have been considered, we conclude that $m_{j}$ is a join prime element of $\operatorname{SFL}(n)$.

Next, we are going to show that $m_{j}$ is meet prime in $\operatorname{SFL}(n)$. Suppose the contrary, and pick $h_{1}, h_{2} \in \mathrm{SFL}(n)$ such that $h_{1} \wedge h_{2} \leq m_{j}$ but $h_{1} \not \leq m_{j}$ 
and $h_{2} \not m_{j}$. We are going to obtain a contradiction by infinite descent. In order to do so, it suffices to show that the condition

$$
j-r \in \mathbb{N}_{0} \text { and there is a } u_{r} \in[n) \text { such that } h_{1} \wedge h_{2} \leq p_{j-r}^{\left(u_{r}\right)}
$$

holds for $r=0$, and to show that

$$
\begin{aligned}
& \text { for every } r \in \mathbb{N}_{0} \text {, if (4.17) holds for } \\
& r \text {, then it also holds for } r+1 \text {. }
\end{aligned}
$$

Applying $(\mathrm{W})$ to $h_{1} \wedge h_{2} \leq m_{j}=\bigvee_{u_{0}<v_{0}}\left(p_{j}^{\left(u_{0}\right)} \wedge p_{j}^{\left(v_{0}\right)}\right)$, we obtain $u_{0}, v_{0} \in[n)$ such that $h_{1} \wedge h_{2} \leq p_{j}^{\left(u_{0}\right)} \wedge p_{j}^{\left(v_{0}\right)} \leq p_{j}^{\left(u_{0}\right)}=p_{j-0}^{\left(u_{0}\right)}$. Hence, (4.17) holds for $r=0$. Next, in order to show (4.18), assume that $r \in \mathbb{N}_{0}$ satisfies condition (4.17). We cannot have that $h_{1} \wedge h_{2} \leq x_{u_{r}}$, because otherwise $h_{i} \leq x_{u_{r}}$ for some $i \in\{1,2\}$ by the meet primeness of $x_{u_{r}}$, see Lemma 4.1, and so (4.2) would give that $h_{i}=0_{\mathrm{SFL}(n)} \leq m_{j}$, contradicting our assumption. Combining $h_{1} \wedge h_{2} \not \leq x_{u_{r}}$ with (4.17) and (3.2), we obtain that $j-r \neq 0$. Hence, $j-(r+1) \in \mathbb{N}_{0}$ and

$$
h_{1} \wedge h_{2} \leq p_{j-r}^{\left(u_{r}\right)}=x_{u_{r}} \vee \bigvee_{u_{r+1}<v_{r+1}}\left(p_{j-(r+1)}^{\left(u_{r+1}\right)} \wedge p_{j-(r+1)}^{\left(v_{r+1}\right)}\right) .
$$

In order to exclude that $h_{1} \leq p_{j-r}^{\left(u_{r}\right)}$, suppose the contrary. So, by Lemma 4.6, $h_{1} \leq p_{j-r}^{\left(u_{r}\right)} \leq p_{j}^{\left(u_{r}\right)}$. Using a permutation of $[n)$ with $u_{r} \mapsto v_{r} \in[n) \backslash\left\{u_{r}\right\}$ as in (4.16), we obtain that $h_{1} \leq p_{j}^{\left(v_{r}\right)}$. Hence, $h_{1} \leq p_{j}^{\left(u_{r}\right)} \wedge p_{j}^{\left(v_{r}\right)} \leq m_{j}$ is a contradiction, proving that $h_{1} \not \leq p_{j-r}^{\left(u_{r}\right)}$. Similarly, $h_{2} \nless \leq p_{j-r}^{\left(u_{r}\right)}$. Now that we have seen that $h_{1} \wedge h_{2} \not \leq x_{u_{r}}, h_{1} \not \leq p_{j-r}^{\left(u_{r}\right)}$, and $h_{2} \not \leq p_{j-r}^{\left(u_{r}\right)}$, we are in the position to apply (W) to (4.19). So we obtain that $h_{1} \wedge h_{2} \leq p_{j-(r+1)}^{\left(u_{r+1}\right)} \wedge p_{j-(r+1)}^{\left(v_{r+1}\right)}$ for some $u_{r+1}, v_{r+1} \in[n)$. Hence, $h_{1} \wedge h_{2} \leq p_{j-(r+1)}^{\left(u_{r+1}\right)}$ and (4.17) holds for $r+1$. We have verified (4.18). Thus, it follows that (4.17) holds for all $r \in \mathbb{N}_{0}$. This is the required contradiction proving that $m_{j}$ is meet prime in $\operatorname{SFL}(n)$, completing the proof of Lemma 4.8 .

For $3 \leq n \in \mathbb{N}^{+}$, in connection with (3.2) and Lemma 4.5, let

$$
P_{n}:= \begin{cases}\left\{m_{j} \in \operatorname{SFL}(n): j \in \mathbb{N}^{+}\right\} \cup\left\{\overline{m_{j}} \in \operatorname{SFL}(n): j \in \mathbb{N}^{+}\right\}, & \text {if } n=3 \\ \left\{m_{j} \in \operatorname{SFL}(n): j \in \mathbb{N}_{0}\right\} \cup\left\{\overline{m_{j}} \in \operatorname{SFL}(n): j \in \mathbb{N}_{0}\right\}, & \text { if } n>3 .\end{cases}
$$

With the ordering of $\operatorname{SFL}(n)$ restricted to $P_{n}, \mathbf{P}_{n}=\left(P_{n} ; \leq\right)$ is an ordered set, which is described by the following lemma; see also Figure 1 for $3<n \in \mathbb{N}^{+}$. This lemma explains why the case $n=3$ differs slightly from the case $n>3$.

Lemma 4.9. The following four assertions hold.

(i) If $n>3$, then $s<m_{0} \not \overline{m_{0}}$.

(ii) If $n=3$, then $m_{1} \not \overline{m_{1}}$. However, $m_{0}=s<\bar{s}=\overline{m_{0}}$, so $m_{0}$ and $\overline{m_{0}}$ are the unique atom and the unique coatom of $\mathrm{SFL}(3)$, respectively.

(iii) For $n \geq 3$ and $i, j \in \mathbb{N}^{+}, m_{i} \not \leq \overline{m_{j}}$.

(iv) For $n \geq 3$ and $i, j \in \mathbb{N}_{0}, \overline{m_{j}} \not \leq m_{i}$. 
Proof. If $n>3$, then letting $\vec{w}:=(1,1,0, \ldots, 0)$, we have that $s(\vec{w})=0$, $m_{0}(\vec{w})=1$, and $\overline{m_{0}}(\vec{w})=0$ hold in the two-element lattice 2 . This proves (i), because $s \leq m_{0}$ follows from Lemma 4.5.

In addition to a straightforward calculation, which will be omitted, (ii) has also been checked by the computer program of Section 7 .

Next, to deal with (ii), we assume that $n=3$. Clearly, $m_{0}=s$ and $\overline{m_{0}}=\bar{s}$. Lemma 4.5 gives that $s \leq \bar{s}$ while $\mathrm{M}_{3}$ witnesses that $s \neq \bar{s}$. We have seen that $m_{0}=s<\bar{s}=\overline{m_{0}}$. For the sake of contradiction, suppose that $m_{1} \leq \overline{m_{1}}$. Then each of the joinands of $m_{1}$ is less than or equal to every meetand of $\overline{m_{1}}$. In particular, we have that $p_{1}^{(0)} \wedge p_{1}^{(1)}$ is less than or equal to its dual, that is,

$$
\begin{aligned}
& \left(x_{0} \vee\left(x_{1} \wedge x_{2}\right)\right) \wedge\left(x_{1} \vee\left(x_{0} \wedge x_{2}\right)\right) \\
& \quad \leq\left(x_{0} \wedge\left(x_{1} \vee x_{2}\right)\right) \vee\left(x_{1} \wedge\left(x_{0} \vee x_{2}\right)\right) .
\end{aligned}
$$

By $(\mathrm{W})$, duality, and since $x_{0}$ and $x_{1}$ play symmetric roles, we can assume that (4.20) holds after omitting its underlined meetand. Hence, $x_{0}$ is less than or equal to the right hand side of (4.20). Using that $x_{0}$ is join prime by Lemma 4.1, we obtain that either $x_{0} \leq x_{0} \wedge\left(x_{1} \vee x_{2}\right) \leq x_{1} \vee x_{2}$, or $x_{0} \leq x_{1} \wedge\left(x_{0} \vee x_{2}\right) \leq x_{1}$, so we have obtained a contradiction, proving (ii).

Next, we turn our attention to (iii). Suppose, for a contradiction, that $m_{i} \leq \overline{m_{j}}$ for some $i, j \in \mathbb{N}^{+}$. We obtain from Lemma 4.7 that

$$
m_{0}<m_{1} \leq m_{i} \leq \overline{m_{j}} \leq \overline{m_{1}}<\overline{m_{0}} .
$$

In particular, $m_{0}<\overline{m_{0}}$ and $m_{1} \leq \overline{m_{1}}$. The first of these two inequalities contradicts part (i) if $n>3$, while the second one contradicts part (ii) if $n=3$. So we obtain a contradiction for all $n \geq 3$, whereby (iii) holds.

Finally, we obtain (iv) basically from $\eta\left(\overline{m_{j}}\right)=1$ and $\eta\left(m_{i}\right)=0$, see (4.3), as follows. Using (4.5), its dual, and (3.2) defining our terms, we obtain that $\overline{m_{j}}\left(a_{0}, \ldots, a_{n-1}\right)=1_{\mathrm{M}_{n}}$ and $m_{i}\left(a_{0}, \ldots, a_{n-1}\right)=0_{\mathrm{M}_{n}}$. This implies (iv) and completes the proof of Lemma 4.9 .

Now, to indicate that we are progressing in the desired direction, we are going to formulate a corollary. Note, however, that neither this corollary, nor its proof, nor the concept defined in the present paragraph will be used in this paper, so the reader can skip over them. Following Dean [3] and Dilworth [5], an ordered set $P=\left(P ; \leq_{P}\right)$ completely freely generates a lattice $K$ if $P$ is a subset of $K, \leq_{P}$ is the restriction of the lattice order $\leq_{K}$ to $P$, and for every lattice $L$ and every order-preserving map $\varphi:\left(P ; \leq_{P}\right) \rightarrow L$, there exists a lattice homomorphism $K \rightarrow L$ that extends $\varphi$. If so, then we denote $K$ by $\mathrm{CF}(P ; \leq)$. The ordered set $\left(P_{n} ; \leq\right)$ was defined right before (and in) Lemma 4.9 ; let $\left[P_{n}\right]_{\mathrm{FL}(n)}=\left[P_{n}\right]_{\mathrm{SFL}(n)}$ denote the sublattice generated by it.

Corollary 4.10. (i) As an ordered set, $\left(P_{n} ; \leq\right)$ is described by Lemmas 4.7 and 4.9 ; note that for $3<n \in \mathbb{N}^{+},\left(P_{n} ; \leq\right)$ is given also by Figure 1 .

(ii) The sublattice $\left[P_{n}\right]_{\mathrm{FL}(n)}=\left[P_{n}\right]_{\mathrm{SFL}(n)}$ is completely freely generated by $\left(P_{n} ; \leq\right)$.

(iii) Furthermore, $\operatorname{SFL}(n)$ has a sublattice isomorphic to $\mathrm{FL}(\omega)$. 
Part (iii) is a consequence of Theorem 1.2(B); the point is that we can easily conclude Corollary 4.10(iii) from known results and the previous lemmas.

Proof of Corollary 4.10. As opposed to other proofs in the paper, the present argument relies on some outer references that are not quoted with full details. Part (i) is clear. For the validity of Part (ii), we need to show that for arbitrary $(k+k)$-ary lattice terms $t_{1}$ and $t_{2}$, the inequality

$$
t_{1}\left(m_{i}: i<k, \overline{m_{i}}: i<k\right) \leq t_{2}\left(m_{i}: i<k, \overline{m_{i}}: i<k\right)
$$

holds in $\mathrm{FL}(n)$ iff it holds in the completely free lattice $\mathrm{CF}\left(P_{n} ; \leq\right)$. The satisfaction of (4.21) in $\mathrm{CF}\left(P_{n} ; \leq\right)$ can be tested by Dean's algorithm, which is a generalization of Whitman's algorithm; see Dean [3] or see Freese, Ježek, and Nation [9, Theorem 5.19]. This is a recursive algorithm that uses only the following three properties of $\mathrm{CF}\left(P_{n} ; \leq\right)$ and $\left(P_{n} ; \leq\right)$ :

(D1) $\quad \mathrm{CF}\left(P_{n} ; \leq\right)$ satisfies $(\mathrm{W})$,

(D2) the elements of $P_{n}$ are doubly prime, and

(D3) the description of the ordering of $P_{n}$.

It follows from Lemmas 4.2, 4.7, 4.8, and 4.9 that these properties hold for $P_{n}$ as a subset of $\mathrm{FL}(n)$. Therefore, Dean's algorithm gives the same result in $\mathrm{CF}\left(P_{n} ; \leq\right)$ as $(\mathrm{D} 1)-(\mathrm{D} 3)$ give in $\mathrm{FL}(n)$. Hence, we conclude that $\left[P_{n}\right]_{\mathrm{FL}(n)}$ is completely freely generated by $\left(P_{n} ; \leq\right)$, proving Part (ii). Thus, $\operatorname{CF}\left(P_{n} ; \leq\right)$ can be embedded into $\operatorname{SFL}(n)$. Using that $\mathrm{FL}(\omega)$ can be embedded into $\mathrm{FL}(3)$ by Whitman [20] and $\mathrm{FL}(3)$ can be embedded into $\mathrm{CF}\left(P_{n} ; \leq\right)$ by the main result of Rival and Wille [17], we conclude by transitivity that $\operatorname{SFL}(n)$ has a sublattice isomorphic to $\mathrm{FL}(\omega)$. This proves Part (iii).

Now, we are ready to prove our Key Lemma.

Proof of Lemma 3.1. It is clear by (3.2) that

$$
\left\{m_{j}: j \in \mathbb{N}_{0}\right\} \cup\left\{\overline{m_{j}}: j \in \mathbb{N}_{0}\right\} \cup\{a, b, \bar{a}, \bar{b}\} \subseteq \operatorname{SFL}(n) .
$$

In order to apply Lemma 4.3 and complete the proof in this way, it suffices to show that none of the inequalities

$\begin{array}{ll}\text { (ineq1) } & x_{0} \leq a \vee \bar{a} \vee b \vee \bar{b}, \\ \text { (ineq2) } & a \leq x_{0} \vee \bar{a} \vee b \vee \bar{b}, \\ \text { (ineq3) } & b \leq x_{0} \vee a \vee \bar{a} \vee \bar{b}, \\ \text { (ineq4) } & \bar{a} \leq x_{0} \vee a \vee b \vee \bar{b}, \text { and } \\ \text { (ineq5) } & \bar{b} \leq x_{0} \vee a \vee \bar{a} \vee b\end{array}$

holds in $\mathrm{FL}(n)$, because then the same will be true for their duals. For example, if $a \geq x_{0} \wedge \bar{a} \wedge b \wedge \bar{b}$ held, then we could apply $\delta=\delta_{\mathrm{FL}(n)}$ from (1.1) to this inequality to obtain that (ineq4) holds.

First, we consider (ineq1). Suppose, for a contradiction, that it holds. Using (4.22), the elements we are going to deal with are in $\operatorname{SFL}(n)$. For $i \in \mathbb{N}^{+}$, we have that $m_{i}<m_{i+1}$ by Lemma 4.7 , whereby Lemma 4.5 gives that $m_{i} \leq \bar{s}$; see Figure 1 for the meaning of $\bar{s}$ (and that of $s$ ). Since $m_{0}<m_{i}$ by Lemma 4.7, 
Lemma 4.5 gives that $s \leq m_{i}$, whence $\overline{m_{i}} \leq \bar{s}$. Since $m_{i} \leq \bar{s}$ and $\overline{m_{i}} \leq \bar{s}$ for all $i \in \mathbb{N}^{+},(3.2)$ gives that $a \vee \bar{a} \vee b \vee \bar{b} \leq \bar{s}$. This is a contradiction, because (4.2) and (ineq1) imply that $a \vee \bar{a} \vee b \vee \bar{b}=1_{\mathrm{SFL}(n)}>\bar{s}$.

Second, for the sake of contradiction, suppose that (ineq2) holds. Since $\overline{m_{3}} \leq a$, we obtain that

$$
\overline{m_{3}} \leq x_{0} \vee\left(\overline{m_{1}} \wedge m_{3}\right) \vee m_{2} \vee \overline{m_{4}} \vee\left(\overline{m_{2}} \wedge m_{4}\right) .
$$

By Lemma 4.7, $\overline{m_{3}} \nless \overline{m_{4}}$. None of the inequalities $\overline{m_{3}} \leq \overline{m_{1}} \wedge m_{3} \leq m_{3}$, $\overline{m_{3}} \leq m_{2}$, and $\overline{m_{3}} \leq \overline{m_{2}} \wedge m_{4} \leq m_{4}$ holds by Lemma 4.9 (iv). Thus, since $\overline{m_{3}}$ is join prime by Lemma 4.8, it follows from (4.23) that $\overline{m_{3}} \leq x_{0}$. Hence, (4.2) yields that $\overline{m_{3}}=0_{\mathrm{SFL}(n)}$. So $0_{\mathrm{SFL}(n)}=\overline{m_{3}}>\overline{m_{4}} \in \operatorname{SFL}(n)$ by Lemma 4.7, and this is a contradiction. Thus, (ineq2) fails, as required.

Third, for the sake of contradiction, we suppose that (ineq3) holds. Since $m_{2} \leq b$, we obtain that

$$
m_{2} \leq x_{0} \vee m_{1} \vee \overline{m_{3}} \vee\left(\overline{m_{1}} \wedge m_{3}\right) \vee\left(\overline{m_{2}} \wedge m_{4}\right) .
$$

By Lemma 4.7, $m_{2} \nless m_{1}$. None of the inequalities $m_{2} \leq \overline{m_{1}} \wedge m_{3} \leq \overline{m_{1}}$, $m_{2} \leq \overline{m_{3}}$, and $m_{2} \leq \overline{m_{2}} \wedge m_{4} \leq \overline{m_{2}}$ holds by Lemma 4.9 (iii). Therefore, since $m_{2}$ is join prime by Lemma 4.8, (4.24) gives that $m_{2} \leq x_{0}$. Hence, (4.2) and Lemma 4.7 yield that $m_{1}<m_{2}=0_{\mathrm{SFL}(n)}$, contradicting $m_{1} \in \operatorname{SFL}(n)$. Therefore, (ineq3) fails, as required.

Clearly, there is a lot of similarity between the treatment for (ineq2) and that for (ineq3). Namely, both arguments rely on (4.2), Lemmas 4.7, 4.8, and 4.9 , and some comparabilities among the subscripts. In an analogous way, the argument for (ineq4) and that for (ineq5) are also very similar; this justifies that only the first of them will be detailed.

For the sake of contradiction, suppose that (ineq4) is satisfied, that is,

$$
\overline{m_{1}} \wedge m_{3} \leq x_{0} \vee m_{1} \vee \overline{m_{3}} \vee m_{2} \vee \overline{m_{4}} \vee \underline{\left(\overline{m_{2}} \wedge m_{4}\right)}
$$

We are going to parse this inequality by (W), taking into account that, according to Lemmas 4.1 and 4.8 , both meetands on the left and the five nonunderlined joinands on the right of (4.25) are doubly prime elements. Therefore, either one of the two meetands is less than or equal to one of the five non-underlined joinands, or $\overline{m_{1}} \wedge m_{3} \leq \overline{m_{2}} \wedge m_{4}$; so we need to consider only these possibilities. If we had that $\overline{m_{1}} \leq x_{0}$ or $m_{3} \leq x_{0}$, then (4.2) and Lemma 4.7 would lead to $\overline{m_{2}}<\overline{m_{1}}=0_{\mathrm{SFL}(n)}$ or $m_{2}<m_{3}=0_{\mathrm{SFL}(n)}$, which are contradictions. If one of the two meetands was less than or equal to another non-underlined joinand, then Lemma 4.7 or Lemma 4.9 would prompt give a contradiction. We are left with the case $\overline{m_{1}} \wedge m_{3} \leq \overline{m_{2}} \wedge m_{4}$, but then $\overline{m_{1}} \wedge m_{3} \leq \overline{m_{2}}$, so the meet primeness of $\overline{m_{2}}$ gives that $\overline{m_{1}} \leq \overline{m_{2}}$, contradicting Lemma 4.7, or $m_{3} \leq \overline{m_{2}}$, contradicting Lemma 4.9(iii). Therefore, (ineq4) fails, as required. Finally, as we have already mentioned, (ineq5) fails by an analogous argument. This completes the proof of Lemma 3.1. 


\section{From the Key Lemma to a stronger statement}

If a subset $X$ of a lattice freely generates, then so do the subsets of $X$. Thus, (the Key) Lemma 3.1 implies that, for every $3 \leq \lambda \in \mathbb{N}^{+}$, there is a totally symmetric embedding $\mathrm{FL}(4) \rightarrow \mathrm{FL}(\lambda)$. In particular, Lemma 3.1 implies Corollary 1.3. In this section, with the extensive help of Czédli [2], we lift the rank 4 of $\mathrm{FL}(4)$ to all even natural numbers $\kappa \geq 4$ and even to $\aleph_{0}$. That is, we are going to prove the following lemma. Remember that $a, \bar{a}, b$ and $\bar{b}$ have been defined in (3.2).

Lemma 5.1. If $\kappa=\aleph_{0}$ or $\kappa \geq 4$ is an even integer, then for every integer $\lambda=$ $n \geq 3$, there exists a totally symmetric embedding $\tau_{\kappa \lambda}: \mathrm{FL}(\kappa) \rightarrow \mathrm{FL}(\lambda)$ with the additional property that $\tau_{\kappa \lambda}(\mathrm{FL}(\kappa))$ is included in the sublattice generated by $\{a, \bar{a}, b, \bar{b}\}$.

Proof. First, in order to make our references to Czédli [2] convenient, we need to deal with the notation. Let $\left(y_{1}, y_{2}, y_{3}, y_{4}\right):=(a, \bar{a}, b, \bar{b}) \in \operatorname{SFL}(n)^{4}$. It follows from (the Key) Lemma 3.1 that $\left\{y_{1}, \ldots, y_{4}\right\}$ freely generates. This allows us to write $\mathrm{FL}(4)=\mathrm{FL}\left(y_{1}, \ldots, y_{4}\right)$ in the present proof, so $\mathrm{FL}(4)$ is a sublattice of $\operatorname{SFL}(n)$. Since $\left\{y_{1}, \ldots, y_{4}\right\}=\{a, \bar{a}, b, \bar{b}\}$ is closed with respect to $\delta_{\mathrm{FL}(n)}$ defined in $(1.1), \mathrm{FL}(4)=\mathrm{FL}\left(y_{1}, \ldots, y_{4}\right)$ is selfdually positioned in $\mathrm{FL}(n)$. Hence,

$$
\text { the restriction } \left.\delta_{4}^{\mathrm{sw}}:=\delta_{\mathrm{FL}(n)}\right]_{\mathrm{FL}(4)} \text { of } \delta_{\mathrm{FL}(n)} \text { to } \mathrm{FL}(4) \text {, }
$$

is a dual automorphism of FL(4). Note the rule that

$$
\delta_{4}^{\mathrm{sw}}\left(y_{1}\right)=y_{2}, \quad \delta_{4}^{\mathrm{sw}}\left(y_{2}\right)=y_{1}, \quad \delta_{4}^{\mathrm{sw}}\left(y_{3}\right)=y_{4}, \quad \delta_{4}^{\mathrm{sw}}\left(y_{4}\right)=y_{3} .
$$

We do not need the exact definition of the lattice terms $x_{1}^{1+i}$ and $x_{2}^{1+i}$ given in [2, Section 4], but we have to recall some of their properties. For $i \in \mathbb{N}_{0}, x_{1}^{1+i}$ and $x_{2}^{1+i}$ are lattice terms over $\left\{y_{1}, \ldots, y_{4}\right\}$, that is, they belong to $\mathrm{FL}(4)$. For brevity, we denote $\left\{x_{1}^{1+i}: 2 i<\kappa\right\} \cup\left\{x_{2}^{1+i}: 2 i<\kappa\right\}$ by $\left\{x_{1}^{1+i}, x_{2}^{1+i}: 2 i<\kappa\right\}$. By [2, Lemma 4.1], $\left\{x_{1}^{1+i}, x_{2}^{1+i}: 2 i<\kappa\right\}$ freely generates a sublattice of FL(4). Hence, in the present proof, we can write that

$$
\mathrm{FL}(\kappa)=\mathrm{FL}\left(x_{1}^{1+i}, x_{2}^{1+i}: 2 i<\kappa\right)=\left[x_{1}^{1+i}, x_{2}^{1+i}: 2 i<\kappa\right]_{\mathrm{FL}(4)} .
$$

For example, $\mathrm{FL}(6)=\mathrm{FL}\left(x_{1}^{1+i}, x_{2}^{1+i}: 2 i<6\right)=\mathrm{FL}\left(x_{1}^{1}, x_{2}^{1}, x_{1}^{2}, x_{2}^{2}, x_{1}^{3}, x_{2}^{3}\right)$. It is important that 6 and, in general, $\kappa$ is even or $\aleph_{0}$, because for an odd integer $\kappa \in \mathbb{N}^{+}, \mathrm{FL}\left(x_{1}^{1+i}, x_{2}^{1+i}: 2 i<\kappa\right)$ would be $\mathrm{FL}(\kappa+1)$ rather than $\mathrm{FL}(\kappa)$.

This paragraph is to tailor the second half of [2, Lemma 4.1] to the present situation; the reader may want to skip over it. It is irrelevant for us what $a$ and $b$ denote in [2]; they are not the same as here. It is also irrelevant that $\mathrm{FL}(4)=\mathrm{FL}\left(y_{1}, \ldots, y_{4}\right)$ is embedded into $\mathrm{FL}(x, y, z)$ in [2] but into $\mathrm{FL}(n)$ here; these two embeddings are different even for $n=3$. Using the first page, Lemma 2.1(B), and the second line of Section 4 of [2], we obtain from [2] that $\left.\delta=\delta_{\mathrm{FL}(x, y, z)}\right]_{\mathrm{FL}\left(y_{1}, \ldots, y_{4}\right)}$ in $[2$, Lemma 4.1] denotes a dual automorphism of $\mathrm{FL}(4)=\mathrm{FL}\left(y_{1}, \ldots, y_{4}\right)$ such that $(5.2)$ holds also for $\delta$. Therefore, $\delta$ in $[2$, Lemma 4.1] is the same as $\delta_{4}^{\text {sw }}$ here, and the second half of [2, Lemma 4.1] asserts that $\delta_{4}^{\mathrm{sw}}\left(x_{1}^{i}\right)=x_{2}^{i}$ and $\delta_{4}^{\mathrm{sw}}\left(x_{2}^{i}\right)=x_{1}^{i}$ for all meaningful $i$. 
So, [2, Lemma 4.1] yields that $\delta_{4}^{\mathrm{sw}}\left(x_{1}^{i}\right)=x_{2}^{i}$ and $\delta_{4}^{\mathrm{sw}}\left(x_{2}^{i}\right)=x_{1}^{i}$ hold for all $i$ such that $2 i<\kappa$. Therefore, since $\delta_{4}^{\text {sw }}$ is the restriction of $\delta_{\mathrm{FL}(n)}$ by (5.1), the set $\left\{\left(x_{1}^{1+i}, x_{2}^{1+i}: 2 i<\kappa\right\}\right.$ is selfdually positioned in $\operatorname{FL}(n)$. Consequently, so is the sublattice $\operatorname{FL}(\kappa)=\left[x_{1}^{1+i}, x_{2}^{1+i}: 2 i<\kappa\right]_{\mathrm{FL}(n)}$. Finally, $\mathrm{FL}(\kappa) \subseteq\left[y_{1}, \ldots, y_{4}\right]_{\mathrm{FL}(n)}=[a, \bar{a}, b, \bar{b}]_{\mathrm{FL}(n)} \subseteq \operatorname{SFL}(n)$. Hence, the inclusion map $\tau_{\kappa \lambda}: \mathrm{FL}(\kappa) \rightarrow \mathrm{FL}(n)=\mathrm{FL}(\lambda)$ is a totally symmetric embedding and $\tau_{\kappa \lambda}(\mathrm{FL}(\kappa)) \subseteq[a, \bar{a}, b, \bar{b}]$. This completes the proof of Lemma 5.1.

\section{The rest of the proofs}

Proof of Theorem 1.4. If $\mathrm{FL}(\lambda)$ has a selfdually positioned sublattice isomorphic to $\mathrm{FL}(\kappa)$, then $\max \left\{\kappa, \aleph_{0}\right\}=|\mathrm{FL}(\kappa)| \leq|\mathrm{FL}(\lambda)|=\max \left\{\lambda, \aleph_{0}\right\}$, as required. Conversely, assume that $\max \left\{\kappa, \aleph_{0}\right\} \leq \max \left\{\lambda, \aleph_{0}\right\}$. To specify the free generators, we let $\mathrm{FL}(\kappa)=\mathrm{FL}\left(y_{i}: i<\kappa\right)$ and $\mathrm{FL}(\lambda)=\operatorname{FL}\left(x_{i}: i<\lambda\right)$. We can assume that $\kappa>\lambda$, since otherwise the sublattice $\left[x_{i}: i<\kappa\right]$ generated by $\left\{x_{i}: i<\kappa\right\}$ in $\mathrm{FL}(\lambda)$ is selfdually positioned and it is isomorphic to $\mathrm{FL}(\kappa)$. The inequality $\kappa>\lambda$ together with $\max \left\{\kappa, \aleph_{0}\right\} \leq \max \left\{\lambda, \aleph_{0}\right\}$ and $3 \leq \kappa$ give that $4 \leq \kappa \leq \aleph_{0}$ and $\lambda \in \mathbb{N}^{+}$. We can assume that $\kappa=2 k+1 \geq 4$ is an odd integer, since otherwise Lemma 5.1 gives a totally symmetric embedding $\varphi: \mathrm{FL}(\kappa) \rightarrow \mathrm{FL}(\lambda)$ and $\varphi(\mathrm{FL}(\kappa))$ does the job. Again by Lemma 5.1, we can pick a $2 k$-element subset $C=\left\{c_{i}: i<2 k\right\}$ of the sublattice $[a, \bar{a}, b, \bar{b}]$ of $\operatorname{FL}(\lambda)$ such that the sublattice $[C]$ is selfdually positioned in $\operatorname{FL}(\lambda)$ and $[C]$ is freely generated by $C=\left\{c_{i}: i<2 k\right\}$. Since the natural dual automorphism $\delta_{\mathrm{FL}(\lambda)}$ from (1.1) preserves double irreducibility in $[C]$, it follows, after slight notational changes, from (2.1) and (2.3) that the set $C$ itself is selfdually positioned, that is, $\delta_{\mathrm{FL}(\lambda)}(C)=C$. Let $D=C \cup\left\{x_{0}\right\}$. Since $\delta_{\mathrm{FL}(\lambda)}\left(x_{0}\right)=x_{0}$ by definition, $\delta_{\mathrm{FL}(\lambda)}(D)=D$. This yields that $[D]=[D]_{\mathrm{FL}(\lambda)}$, the sublattice generated by $D$, is selfdually positioned, that is, $\delta_{\mathrm{FL}(\lambda)}([D])=[D]$. Therefore, since $|D|=2 k+1=\kappa$, we need to show only that $D$ freely generates. By duality and Lemma 4.3, it suffices to exclude that

$$
\begin{aligned}
& x_{0} \leq c_{0} \vee c_{1} \vee \cdots \vee c_{2 k-1}, \quad \text { or } \\
& c_{j} \leq x_{0} \vee \bigvee_{i \in[2 k) \backslash\{j\}} c_{i} \quad \text { for some } j \in[2 k) .
\end{aligned}
$$

We know from Lemma 3.1 that the sublattice $S:=\left[a, \bar{a}, b, \bar{b}, x_{0}\right]$ of $\operatorname{FL}(n)$ is freely generated by the set $\left\{a, \bar{a}, b, \bar{b}, x_{0}\right\}$. Therefore, the self-maps

$$
\xi_{1}:=\left(\begin{array}{ccccc}
a & \bar{a} & b & \bar{b} & x_{0} \\
0_{S} & 0_{S} & 0_{S} & 0_{S} & 1_{S}
\end{array}\right) \quad \text { and } \quad \xi_{2}:=\left(\begin{array}{ccccc}
a & \bar{a} & b & \bar{b} & x_{0} \\
a & \bar{a} & b & \bar{b} & 0_{S}
\end{array}\right)
$$

extend to endomorphisms $\widehat{\xi}_{1}: S \rightarrow S$ and $\widehat{\xi}_{2}: S \rightarrow S$, respectively. Using the inclusion $C \subseteq[a, \bar{a}, b, \bar{b}]$, we obtain that $\xi_{1}([a, \bar{a}, b, \bar{b}])=\left\{0_{S}\right\}$. Taking the equality $\xi_{1}\left(x_{0}\right)=1_{S}$ also into account, we obtain that the endomorphism $\widehat{\xi}_{1}$ does not preserve inequality (6.1). Hence, (6.1) fails, as required. Next, suppose that (6.2) holds. Using $C \subseteq[a, \bar{a}, b, \bar{b}]$, it follows that the restriction of $\widehat{\xi}_{2}$ to $[a, \bar{a}, b, \bar{b}]$ is the identity map. Therefore, since $\widehat{\xi}_{2}$ is order-preserving, its 
application to (6.2) yields that $c_{j} \leq \bigvee_{i \in[2 k) \backslash\{j\}} c_{i}$. But this is a contradiction since $C$ freely generates, and we conclude that (6.2) fails, as required.

Proof of Corollary 2.3. In order to prove the implication (ii) $\Rightarrow$ (i), assume that (ii) holds. We can also assume that $\kappa \neq \lambda$ since otherwise the identity map $\mathrm{FL}(\kappa) \rightarrow \mathrm{FL}(\kappa)=\mathrm{FL}(\lambda)$ does the job. As it is pointed out right after (2.4), $[\operatorname{DAut}(\mathrm{FL}(\lambda)): \operatorname{Aut}(\mathrm{FL}(\lambda))]=2$. Hence, with $\delta_{\mathrm{FL}(\lambda)}$ from (1.1),

$$
\operatorname{DAut}(\mathrm{FL}(\lambda))=\operatorname{Aut}(\mathrm{FL}(\lambda)) \cup\left\{\delta_{\mathrm{FL}(\lambda)} \circ \varphi: \varphi \in \operatorname{Aut}(\mathrm{FL}(\lambda))\right\} .
$$

Thus, the embedding given by Lemma 5.1 has a DAut-symmetric range. Hence, (ii) $\Rightarrow$ (i).

Before proving the converse implications, we formulate and verify some observations, some of which will be useful also in the proof of Corollary 2.4 below. This is why instead of assuming DAut-symmetry, we often assume less, the usual symmetry (with respect to automorphisms). Since Aut(FL $(X))$ acts transitively on the set $X$ of free generators, it follows trivially that

if $S$ is a symmetric sublattice of $\mathrm{FL}(X)$ such that $S \cap X \neq \emptyset$, then $S=\mathrm{FL}(\lambda)$.

As a straightforward consequence of (2.1), observe that

$$
\begin{aligned}
& \text { if } \varphi: \mathrm{FL}(\kappa) \rightarrow \mathrm{FL}(\lambda) \text { is an arbitrary embedding } \\
& \text { and } S:=\varphi(\mathrm{FL}(\kappa)) \text {, then }\left|\operatorname{Irr}_{\wedge}^{\vee}(S)\right|=\kappa \text {. }
\end{aligned}
$$

Since automorphisms and dual automorphisms preserve double primeness, we obtain the following observation.

Let $S$ be a symmetric sublattice of $\operatorname{FL}(\lambda)$; then $\tau\rceil_{\operatorname{Irr}_{\wedge}^{\vee}(S)}: \operatorname{Irr}_{\wedge}^{\vee}(S) \rightarrow \operatorname{Irr}_{\wedge}^{\vee}(S)$ is a bijective map for every $\tau \in \operatorname{Aut}(\mathrm{FL}(\lambda))$. If, in addition, $S$ is DAut-symmetric, then

the same holds even for every $\tau \in \operatorname{DAut}(\operatorname{FL}(\lambda))$.

We are going to prove the following property of orbits $\{\tau(u): \tau \in$ $\operatorname{Aut}(\mathrm{FL}(\lambda))\}$ of elements $u \in \mathrm{FL}(\lambda)$.

If $\lambda \geq \aleph_{0}, S$ is a symmetric sublattice of $\mathrm{FL}(\lambda)$, and $u \in S$, then $|S|=\lambda=|\{\tau(u): \tau \in \operatorname{Aut}(\mathrm{FL}(\lambda))\}|$.

In order to show (6.7), let $S$ be a symmetric sublattice of $\mathrm{FL}(\lambda)=\mathrm{FL}(X)$, where $|X|=\lambda$, and let $u \in S$. Obviously, $|S| \leq|\mathrm{FL}(\lambda)|=\lambda$. It is clear by (3.1) that there is a finite subset $Y \subseteq X$ such that $u$ is in the sublattice $[Y]$ generated by $Y$. By the rudiments of cardinal arithmetics, there is a family $\left\{\pi_{i}: i<\lambda\right\}$ of permutations of $X$ such that $\pi_{i}(Y) \cap \pi_{j}(Y)=\emptyset$ for $i \neq j$. Each of these $\pi_{i}$ extends to an automorphism $\pi_{i}^{\text {aut }}$ of $\operatorname{FL}(\lambda)$. If $i \neq j$, then the map

$$
X \rightarrow \mathbf{2}, \quad x \mapsto \begin{cases}1, & \text { if } x \in \pi_{i}(Y), \\ 0, & \text { if } x \notin \pi_{i}(Y), \text { in particular, if } x \in \pi_{j}(Y)\end{cases}
$$

extends to a lattice homomorphism $\mathrm{FL}(\lambda) \rightarrow \mathbf{2}$. Since this homomorphism maps $\pi_{i}^{\text {aut }}(u) \in\left[\pi_{i}(Y)\right]$ and $\pi_{j}^{\text {aut }}(u) \in\left[\pi_{j}(Y)\right]$ to 1 and 0 , respectively, we 
obtain that $\pi_{i}^{\text {aut }}(u) \neq \pi_{j}^{\text {aut }}(u)$. Furthermore, $\{\tau(u): \tau \in \operatorname{Aut}(\operatorname{FL}(\lambda))\} \subseteq S$ since $S$ is a symmetric sublattice. Hence,

$$
\lambda=\left|\left\{\pi_{i}(u): i<\lambda\right\}\right| \leq|\{\tau(u): \tau \in \operatorname{Aut}(\operatorname{FL}(\lambda))\}| \leq|S| \leq \lambda,
$$

which proves (6.7). We also need the following consequence of (6.7).

If $\lambda \geq \aleph_{0}, S$ is a symmetric sublattice of $\operatorname{FL}(\lambda)$, and $\operatorname{Irr}_{\wedge}^{\vee}(S) \neq \emptyset$, then $\left|\operatorname{Irr}_{\wedge}^{\vee}(S)\right|=\lambda$.

In order to show this, let $u \in \operatorname{Irr}_{\wedge}^{\vee}(S)$. Since $S$ is symmetric, the restriction $\left.\tau\right\rceil_{S}$ of an automorphism $\tau \in \operatorname{Aut}(\mathrm{FL}(\lambda))$ to $S$ is an automorphism of $S$. Hence, $\tau(u)=\tau\rceil_{S}(u)$ also belongs to $\operatorname{Irr}_{\wedge}^{\vee}(S)$. Thus, we conclude from (6.7) that $\lambda \leq\left|\operatorname{Irr}_{\wedge}^{\vee}(S)\right| \leq|\operatorname{FL}(\lambda)|=\lambda$, implying the validity of (6.8). In the observation below, $\delta=\delta_{\mathrm{FL}(\lambda)}$ is the natural dual automorphism introduced in (1.1). An involution on a set $Y$ is a map $Y \rightarrow Y$ whose square is the identity map on $Y$.

If $S$ is a DAut-symmetric sublattice of $\operatorname{FL}(\lambda)$, then the restriction $\left.\delta\right|_{\operatorname{Irr}_{\wedge}^{\vee}(S)}$ of $\delta$ to $\operatorname{Irr}_{\wedge}^{\vee}(S)$ is an involution on $\operatorname{Irr}_{\wedge}^{\vee}(S)$.

Since every restriction of an involution is again an involution, (6.9) follows immediately from (6.6). Next, we are going to prove that

if $\kappa \neq \lambda$ and there is an embedding $\mathrm{FL}(\kappa) \rightarrow \mathrm{FL}(\lambda)$ with

DAut-symmetric range, then $\kappa$ is not an odd integer.

Suppose the contrary, and for an odd $\kappa \in \mathbb{N}^{+}$, let $\varphi: \operatorname{FL}(\kappa) \rightarrow \operatorname{FL}(\lambda)$ with range $S:=\varphi(\mathrm{FL}(\kappa))$ witness the failure of (6.10). We know from (6.9) that $\left.\delta\right|_{\operatorname{Irr}_{\wedge}^{\vee}(S)}$ is an involution on $\operatorname{Irr}_{\wedge}^{\vee}(S)$. If $\left.\delta\right|_{\operatorname{Irr}_{\wedge}^{\vee}(S)}$ has a fixed point $u \in \operatorname{Irr}_{\wedge}^{\vee}(S)$, then $u=\left.\delta\right|_{\operatorname{Irr}_{\wedge}^{\vee}(S)}(u)=\delta(u)$ is one of the free generators of $\operatorname{FL}(\lambda)$ by $(2.3)$, whereby (6.4) gives the equality in $\mathrm{FL}(\kappa) \cong S=\mathrm{FL}(\lambda)$, which implies $\kappa=\lambda$ by (2.1), contradicting $\kappa \neq \lambda$. Hence, $\delta\rceil_{\operatorname{Irr}_{\wedge}(S)}$ has no fixed point. By (6.5), this fixed-point-free involution acts on a $\kappa$-element set. Thus, $\kappa$ is not an odd integer, proving (6.10).

Now, armed with $(6.7),(6.8)$, and (6.10), we are in the position to prove that (i) implies (ii). Assume that (i) holds, and let $\varphi: \mathrm{FL}(\kappa) \rightarrow \mathrm{FL}(\lambda)$ be an embedding with DAut-symmetric range $S:=\varphi(\mathrm{FL}(\kappa))$. We can also assume that $\kappa \neq \lambda$ since there is nothing to prove otherwise. Since $\varphi$ is an embedding, $|\mathrm{FL}(\kappa)| \leq|\mathrm{FL}(\lambda)|$. There are two cases, depending on $\lambda$. First, if $\lambda<\aleph_{0}$, then $\kappa$ is not an odd integer by (6.10) and, furthermore, $|\mathrm{FL}(\kappa)| \leq|\mathrm{FL}(\lambda)|=\aleph_{0}$ gives that $\kappa \leq \aleph_{0}$. Hence, (ii) holds in this case. Second, if $\lambda \geq \aleph_{0}$, then

$$
\kappa \stackrel{(2.1)}{=}\left|\operatorname{Irr}_{\wedge}^{\vee}(\mathrm{FL}(\kappa))\right|=\left|\operatorname{Irr}_{\wedge}^{\vee}(S)\right| \stackrel{(6.8)}{=} \lambda,
$$

and (ii) holds again. The proof of Corollary 2.3 is complete.

Proof of Theorem 1.2. It suffices to prove part (A), since it implies part (B). Let $3 \leq \lambda \in \mathbb{N}^{+}$, and assume that $\kappa=\aleph_{0}$ or $3 \leq \kappa \in \mathbb{N}^{+}$is even. Then there exists a totally symmetric embedding from $\mathrm{FL}(\kappa)$ to $\mathrm{FL}(\lambda)$ by Lemma 5.1 .

Conversely, assume that $3 \leq \kappa, 3 \leq \lambda$, and there exists a totally symmetric embedding $\varphi: \mathrm{FL}(\kappa) \rightarrow \mathrm{FL}(\lambda)$. Let $S=\varphi(\mathrm{FL}(\kappa))$ denote the range of $\varphi$; it consists of some symmetric elements of $\operatorname{FL}(\lambda)$. It follows that $\lambda<\aleph_{0}$, because otherwise there would be no symmetric element in $\operatorname{FL}(\lambda)$. Thus, we have also 
that $\kappa \leq \aleph_{0}$, because $\kappa \leq|\mathrm{FL}(\kappa)| \leq|\mathrm{FL}(\lambda)|=\aleph_{0}$. Since $S$ is invariant under the natural dual automorphism $\delta:=\delta_{\mathrm{FL}(\lambda)}$ and it is symmetric, even elementwise symmetric, (6.3) shows that $S$ is DAut-symmetric. By $(6.9),\left.\delta\right|_{\operatorname{Irr}_{\wedge}^{\vee}(S)}$ is an involution on $\operatorname{Irr}_{\wedge}^{\vee}(S)$. No free generator of $\operatorname{FL}(\lambda)$ is a symmetric element of $\operatorname{FL}(\lambda)$, whereby $S$ is disjoint from the set of free generators of $\operatorname{FL}(\lambda)$. If $\left.\delta\right|_{\operatorname{Irr}_{\wedge}^{\vee}(S)}$ had a fixed point $u$, then $u$ would be a fixed point of $\delta$, so (2.3) would imply that $u \in \operatorname{Irr}_{\wedge}^{\vee}(S) \subseteq S$ is a free generator of $\mathrm{FL}(\lambda)$, contradicting the above-mentioned disjointness. Thus, $\delta\rceil_{\operatorname{Irr}_{\wedge}(S)}$ has no fixed point. By (6.5), the fixed-point-free involution $\left.\delta\right|_{\operatorname{Irr}_{\wedge}^{\vee}(S)}$ acts on the $\kappa$-element set $\operatorname{Irr}_{\wedge}^{\vee}(S)$, and we conclude that $\kappa$ is not an odd integer. That is, $\kappa=\aleph_{0}$ or $\kappa \in \mathbb{N}^{+}$is even, completing the proof.

Proof of Corollary 2.4. In order to prove the "if" part, we can assume that $\kappa \neq \lambda$ since otherwise the identity map of $\mathrm{FL}(\kappa)$ is a required embedding. So $3 \leq \lambda \in \mathbb{N}^{+}$and $3 \leq \kappa \leq \aleph_{0}$. By Theorem 1.2(A), FL $(\lambda)$ has an elementwise symmetric sublattice $S$ such that $S \cong \mathrm{FL}\left(\aleph_{0}\right)$. Since $\kappa \leq \aleph_{0}, \operatorname{FL}(\lambda)$ has also a sublattice $S^{\prime}$ such that $\mathrm{FL}(\kappa) \cong S^{\prime}$. Clearly, any isomorphism from $\mathrm{FL}(\kappa) \rightarrow S^{\prime}$ is an embedding of $\mathrm{FL}(\kappa)$ into $\mathrm{FL}(\lambda)$ with symmetric range; in fact, with element-wise symmetric range. This proves the "if" part.

In order to prove the "only if" part, assume that there is an embedding $\varphi: \operatorname{FL}(\kappa) \rightarrow \mathrm{FL}(\lambda)$ with symmetric range $S$. Clearly, $|\mathrm{FL}(\kappa)| \leq|\mathrm{FL}(\lambda)|$. Depending on $\lambda$, there are two cases to consider. First, if $\lambda<\aleph_{0}$, then $|\operatorname{FL}(\kappa)| \leq|\mathrm{FL}(\lambda)|=\aleph_{0}$ yields that $\kappa \leq \aleph_{0}$, as required. Second, if $\lambda \geq \aleph_{0}$, then (6.11) applies and $\kappa=\lambda$, again as required. The proof is complete.

\section{A computer program and its background}

\section{Historical background}

There are various known algorithms to solve the word problem of free lattices and that of finitely presented lattices. They are discussed in Freese and Nation [11] and in Sections 8 and 9 of Chapter XI of the monograph Freese, Ježek, and Nation [9]; see also Dean [4], Evans [6], McKinsey [13], and Skolem [18] for the original papers. In addition to this list, there is an additional algorithm given in Czédli [1]. We know from [9] that the algorithms given by Skolem, Freese, and Herrmann run in polynomial time; so does the one given in [1]. However, it is only Whitman's algorithm with the modifications explained in [9] that is fast enough for our purposes.

\section{A new computer program}

The first author has developed a Dev-Pascal 1.9.2 (Freepascal) program for the word problem of free lattices. This problem is based on the Freese-Whitman algorithm, as it is given in Freese, Ježek, and Nation [9]. The program runs in Windows environment (tested only under Windows 10), and it can be downloaded from the author's website. The program takes its input from a text file; several sample input files are also donwloadable. We used this program on our personal computer with IntelCore i5-4440 CPU, 3.10 GHz, and 8.00 GB RAM. 


\section{Results achieved with the computer program}

First, we used the program to give alternative proofs. In particular, we used it to prove the second part of the (Key) Lemma 3.1 asserting that $\left\{a, \bar{a}, b, \bar{b}, x_{0}\right\}$ freely generates.

Also, we used the program to prove that

for $n=3$, the Key Lemma remains valid if we replace $m_{1}$,

$m_{2}, m_{3}$ and $m_{4}$ by $m_{5}, m_{7}, m_{8}$, and $m_{9}$, respectively;

this gives an alternative proof of Corollary 1.3. By the paragraph preceding (4.25), it would not be difficult to show that the stipulation $n=3$ can be omitted from (7.2), but this or a similar strengthening of (7.2) is not pursued.

In addition to reaffirming some results from the previous sections, we could use the program to find an entirely new construction to prove Corollary 1.3. In order to describe it, we use the notation introduced in Remark 2.5 to define a join-homomorphism $\nu^{(\mathrm{V})}: \mathrm{FL}(3) \rightarrow \mathrm{SFL}(3)$ and a meet-homomorphism $\mu^{(\wedge)}: \mathrm{FL}(3) \rightarrow \operatorname{SFL}(3)$ by the rules

$$
\nu^{(\vee)}(u):=\bigvee_{\sigma \in \operatorname{Sym}_{3}} \sigma^{\text {aut }}(u) \text { and } \mu^{(\wedge)}(u):=\bigwedge_{\sigma \in \operatorname{Sym}_{3}} \sigma^{\text {aut }}(u) .
$$

In order to ease the notation, we will write $x, y$, and $z$ instead of $x_{0}, x_{1}$, and $x_{2}$, respectively. Note that the program recognizes (appropriate commands for) $\nu^{(\vee)}$ and $\mu^{(\wedge)}$ in input files. Take the following ternary terms, that is, elements of $\mathrm{FL}(3)=\mathrm{FL}(x, y, z)$.

$$
\begin{aligned}
& a_{0}=\nu^{(\vee)}((((x \vee y) \wedge z) \vee y) \wedge(((y \vee x) \wedge z) \vee x)), \\
& a^{\prime}=\mu^{(\wedge)}\left(\left(\left(\left(a_{0} \wedge x\right) \vee y\right) \wedge z\right) \vee\left(((z \wedge x) \vee y) \wedge a_{0}\right)\right), \text { and } \\
& b^{\prime}=\mu^{(\wedge)}\left(\left(\left(((x \vee y) \wedge(x \vee z)) \vee a^{\prime}\right) \wedge x\right) \vee\left(\left(\left(x \wedge a_{0}\right) \vee y\right) \wedge z\right)\right) .
\end{aligned}
$$

With $a^{\prime}$ and $b^{\prime}$ from (7.4) and (7.5) and their duals, $\overline{a^{\prime}}$ and $\overline{b^{\prime}}$, the program proved that

$$
\left\{a^{\prime}, \overline{a^{\prime}}, b, \overline{b^{\prime}}\right\} \text { freely generates a sublattice of } \operatorname{FL}(x, y, z),
$$

which obviously implies Corollary 1.3. Note that for each of (7.1), (7.2), and (7.6), the program ran less than a millisecond on our computer.

Finally, for a lattice term $t$, we define the total number $N_{\text {var }}(t)$ of variables of $t$ by induction as follows: $N_{\mathrm{var}}(t)=1$ if $t$ is a variable and

$$
N_{\text {var }}\left(t_{1} \vee t_{2}\right)=N_{\text {var }}\left(t_{1} \wedge t_{2}\right)=N_{\text {var }}\left(t_{1}\right)+N_{\text {var }}\left(t_{2}\right) .
$$

Note that, say, $x=x \wedge(x \vee y)$ in $\operatorname{FL}(x, y, z)$ but $N_{\text {var }}(x)=1$ is distinct from $N_{\text {var }}(x \wedge(x \vee y))=3$. Hence, as opposed to what (3.1) suggests, we do not define $N_{\text {var }}$ for the elements of $\operatorname{FL}(x, y, z)$. For a set $\left\{t_{1}, \ldots, t_{k}\right\}$ of terms, let $N_{\text {var }}\left(\left\{t_{1}, \ldots, t_{k}\right\}\right)=N_{\text {var }}\left(t_{1}\right)+\cdots+N_{\text {var }}\left(t_{k}\right)$. Table 1 shows how the function $N_{\text {var }}$ compares the terms describing the free generating set given in (3.3) for $n=3$ and those given in (7.4) and (7.6). Another difference between (3.3) and 
TABLE 1. Total number of variables

\begin{tabular}{llll}
\hline & 1st generator & 2nd generator & $N_{\text {var }}($ generating set $)$ \\
\hline$(3.3)_{n=3}$ & $N_{\text {var }}(a)=108$ & $N_{\text {var }}(b)=228$ & $N_{\text {var }}(\{a, \bar{a}, b, \bar{b}\})=672$ \\
$(7.6)$ & $N_{\text {var }}\left(a^{\prime}\right)=612$ & $N_{\text {var }}\left(b^{\prime}\right)=4008$ & $N_{\text {var }}\left(\left\{a^{\prime}, \overline{a^{\prime}}, b^{\prime}, \overline{b^{\prime}}\right\}\right)=9240$ \\
\hline
\end{tabular}

(7.6) is that, as opposed to the set $\left\{a, \bar{a}, b, \bar{b}, x_{0}\right\}$ from (the Key) Lemma 3.1, the program shows that $\left\{a^{\prime}, \overline{a^{\prime}}, b, \overline{b^{\prime}}, x\right\}$ does not generate freely.

\section{Acknowledgements}

Open access funding provided by University of Szeged (SZTE).

Open Access. This article is distributed under the terms of the Creative Commons Attribution 4.0 International License (http://creativecommons.org/licenses/ by $/ 4.0 /$ ), which permits unrestricted use, distribution, and reproduction in any medium, provided you give appropriate credit to the original author(s) and the source, provide a link to the Creative Commons license, and indicate if changes were made.

Publisher's Note Springer Nature remains neutral with regard to jurisdictional claims in published maps and institutional affiliations.

\section{References}

[1] Czédli, G.: On the word problem of lattices with the help of graphs. Period. Math. Hung. 23, 49-58 (1991)

[2] Czédli, G.: A selfdual embedding of the free lattice over countably many generators into the three-generated one. Acta Math. Hung. 148, 100-108 (2016)

[3] Dean, R.A.: Completely free lattices generated by partially ordered sets. Trans. Am. Math. Soc. 83, 238-249 (1956)

[4] Dean, R.A.: Free lattices generated by partially ordered sets and preserving bounds. Can. J. Math. 16, 136-148 (1964)

[5] Dilworth, R.P.: Lattices with unique complements. Trans. Am. Math. Soc. 57, 123-154 (1945)

[6] Evans, T.: The word problem for abstract algebras. Lond. Math. Soc. 26, 64-71 (1951)

[7] Freese, R.: Connected Components of the Covering Relation in Free Lattices. Universal Algebra and Lattice Theory (Charleston, S.C., 1984), Lecture Notes in Math., vol. 1149, pp. 82-93. Springer, Berlin (1985) 
[8] Freese, R.: Free lattice algorithms. Order 3, 331-344 (1987)

[9] Freese, R., Ježek, J., Nation, J. B.: Free Lattices. Mathematical Surveys and Monographs, vol. 42. American Mathematical Society, Providence (1995)

[10] Freese, R., Nation, J.B.: Covers in free lattices. Trans. Am. Math. Soc. 288, 1-42 (1985)

[11] Freese, R., Nation, J. B.: Free and Finitely Presented Lattices. Lattice Theory: Special Topics and Applications, vol. 2, p. 2758. Birkhäuser/Springer, Cham (2016)

[12] Grätzer, G.: Lattice Theory: Foundation. Birkhäuser, Basel (2011)

[13] McKinsey, J.C.C.: The decision problem for some classes of sentences without quantifiers. J. Symb. Logic 8, 61-76 (1943)

[14] Nation, J.B.: Finite sublattices of a free lattice. Trans. Am. Math. Soc. 269, 311-337 (1982)

[15] Nation, J.B.: On partially ordered sets embeddable in a free lattice. Algebra Univers. 18, 327-333 (1984)

[16] Nation, J. B.: Notes on Lattice Theory. http://math.hawaii.edu/ jb/math618/ LTNotes.pdf

[17] Rival, I., Wille, R.: Lattices freely generated by partially ordered sets: which can be "drawn"? J. Reine Angew. Math. 310, 56-80 (1979)

[18] Skolem, T.: Selected Works in Logic. Edited by Jens Erik Fenstad Universitetsforlaget, Oslo, p 732 (1970)

[19] Tschantz, S.T.: Infinite intervals in free lattices. Order 6, 367-388 (1990)

[20] Whitman, P.: Free lattices. Ann. Math. 42, 325-330 (1941)

Gábor Czédli, Gergő Gyenizse and Ádám Kunos

Bolyai Institute

University of Szeged

Aradi vértanúk tere 1

Szeged 6720

Hungary

e-mail [G. Czédli]: czedli@math.u-szeged.hu

URL: http://www.math.u-szeged.hu/ czedli/

e-mail [G. Gyenizse]: gergogyenizse@gmail.com

URL: http://gllrumdsuhg.atw.hu/

e-mail [Á. Kunos]: akunos@math.u-szeged.hu

URL: http://www.math.u-szeged.hu/ akunos/

Received: 8 May 2018.

Accepted: 7 January 2019. 